\title{
ON THE GLOBAL GAN-GROSS-PRASAD CONJECTURE FOR GENERAL SPIN GROUPS
}

\author{
A Dissertation \\ presented to \\ the Faculty of the Graduate School \\ University of Missouri
}

In Partial Fulfillment

of the Requirements for the Degree

Doctor of Philosophy

by

Melissa Emory

Dr. Shuichiro Takeda, Dissertation Supervisor

May 2018 
The undersigned, appointed by the Dean of the Graduate School, have examined the dissertation entitled

\section{ON THE GLOBAL GAN-GROSS-PRASAD CONJECTURE FOR GENERAL SPIN GROUPS}

presented by Melissa Emory,

a candidate for the degree of Doctor of Philosophy of Mathematics, and hereby certify that in their opinion it is worthy of acceptance.

Professor Shuichiro Takeda

Professor William D. Banks

Professor Calin Chindris

Professor Dana Weston

Professor David Retzloff 


\section{ACKNOWLEDGEMENTS}

I would like to thank my advisor Shuichiro Takeda for providing the thesis problem, for his advice, and for answering a plethora of questions. He provided clear explanations and made an intimidating subject accessible to me as someone new to the field.

I am indebted to my husband, Ken Emory, for encouraging me to pursue my education and for his constant love and support. I am especially thankful to my children, Rebecca, Andrew and Kyle, who have always been my greatest supporters and source of immense joy and pride. I am also thankful for my sister Lenaya, for her constant love and encouragement throughout the years.

While in graduate school, I have been treated to amazing professors. In particular, my algebra professors Tom Marley and Calin Chindris and analysis professors Loukas Grafakos, Peter Pivovarov, and Alan Peterson. I am extremely grateful to Dale Cutkosky for his algebraic geometry courses and for answering several questions outside of the classroom. I am beholden to William (Bill) Banks for his willingness to teach a range of number theory topics courses, his constant encouragement, and the many conversations outside of the classroom.

My undergraduate advisor, Griff Elder, encouraged me to pursue a $\mathrm{PhD}$ and I thank him for this. I am especially grateful for the many opportunities he provided while I was his student, as well as his continual advice and encouragement.

I am extremely thankful for my friendships with graduate students, faculty, and staff at Mizzou. In particular, for Jacob Clark, Killian Meehan, Brett Collins, Nick Cox-Stein, Alyssa Cherry, Thomas Polstra, Georgios Ntosidis, and Luiza DeSouza. 
A number of women have provided guidance and direction and have been amazing role models for me to follow, and I am thankful to them. I am especially thankful to Hema Srinivasan, Dana Weston, and Tanya Christiansen for their advice, instruction, and encouragement throughout my time at Mizzou. 


\section{Contents}

Acknowledgements ...................... ii

Abstract .......................... vi

1 Introduction 1

1.1 The Gan-Gross-Prasad Conjecture $\ldots \ldots \ldots \ldots \ldots$

1.1.1 The Original Global Gross-Prasad Conjecture . . . . . . . . 2

1.1.2 The Ichino-Ikeda Refinement . . . . . . . . . . . . . . . . 3

1.2 Outline of Dissertation . . . . . . . . . . . . . . 7

2 Structure of GSpin Groups. $\quad 9$

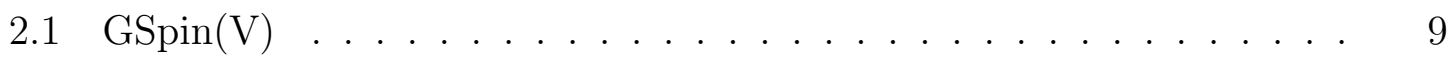

2.2 Root Datum for split GSpin . . . . . . . . . . . . . . . . 13

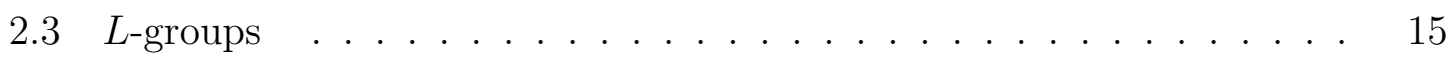

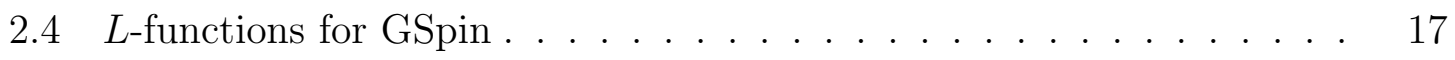

$2.4 .1 \quad L$-functions $\ldots \ldots \ldots \ldots \ldots \ldots \ldots \ldots$

2.4 .2 Tensor product L-functions $\ldots \ldots \ldots \ldots \ldots$

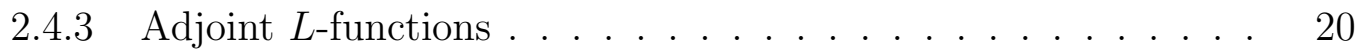

2.5 Component Groups for the local Langlands parameters . . . . . . 20

3 Local Integrals of Matrix Coefficients 23 
3.1 Convergence of the Integral $\ldots \ldots \ldots \ldots$

3.2 Calculation of integrals in the unramified section . . . . . . . 31

3.2.1 Calculation of $\zeta(\Xi, \xi)$ when $E=F \ldots \ldots \ldots$

3.2.2 Calculation of $S_{\Xi^{-1}, \xi^{-1}}$ when $E=F \ldots \ldots \ldots \ldots$

3.2.3 Calculations when $E / F$ is an unramified quadratic extension . 51

4 The Global Gan-Gross-Prasad Conjecture for GSpin $_{2} \times$ GSpin $_{3} \quad 54$

4.1 Waldspurger Formula . . . . . . . . . . . . . . . . . . . . 54

4.1 .1 Linear forms over local fields . . . . . . . . . . . . . . . . 55

4.1 .2 Waldspurger formula . . . . . . . . . . . . . . . 55

4.2 Proof of Conjecture 1.3 for $\mathrm{GSpin}_{2} \times \mathrm{GSpin}_{3}$ :

Waldspurger Formula Case . . . . . . . . . . . . . . 56

4.3 Jacquet-Langlands Case . . . . . . . . . . . . . . . . . . . . 58

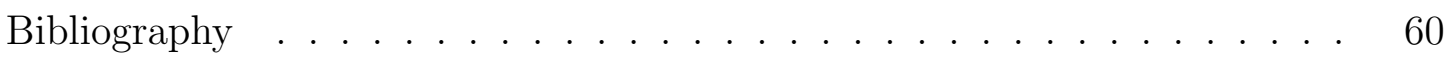

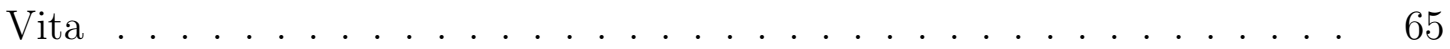


On the global Gan-Gross-Prasad conjecture for general spin groups

\title{
Melissa Emory
}

Dr. Shuichiro Takeda, Dissertation Supervisor

\begin{abstract}
In the 1990s, Benedict Gross and Dipendra Prasad formulated an intriguing conjecture connected with restriction laws for automorphic representations of a particular group. More recently, Gan, Gross, and Prasad extended this conjecture, now known as the Gan-Gross-Prasad Conjecture, to the remaining classical groups. Roughly speaking, they conjectured the non-vanishing of a certain period integral is equivalent to the non-vanishing of the central value of a certain $L$ - function. Ichino and Ikeda refined the conjecture to give an explicit relationship between this central value of a $L$-function and the period integral. We propose a similar conjecture for a nonclassical group, the general spin group, and prove one case.
\end{abstract}




\section{Chapter 1}

\section{Introduction}

In the 1990s, Benedict Gross and Dipendra Prasad [GP92, GP94] formulated an intriguing conjecture regarding the restriction of representations, also known as branching laws, of special orthogonal groups. Restriction problems are one of the most natural problems regarding representations, present from the early days of representation theory. In general, the question is how a representation of a group decomposes when restricted to a subgroup. The Gross-Prasad conjecture describes these restrictions in terms of number theoretic invariants attached to Langlands parameters.

In 2012, Wee Teck Gan, Benedict Gross and Dipendra Prasad [GGP12] extended this conjecture, now known as the Gan-Gross-Prasad (GGP) conjecture, to the remaining classical groups. Roughly speaking, they conjecture that there exists a non-trivial period integral precisely when the central value of the tensor product

$L$-function $L\left(1 / 2, \pi_{n+1} \times \pi_{n}\right)$ is non-zero. Here $\pi_{n+1}$ and $\pi_{n}$ are cuspidal automorphic representations of the groups $\mathrm{SO}\left(V_{n+1}\right)$ and $\mathrm{SO}\left(V_{n}\right)$, where $V_{n+1}$ and $V_{n}$ are quadratic spaces of dimensions $n+1$ and $n$, respectively, such that $V_{n} \subseteq V_{n+1}$.

Atsushi Ichino and Tamotsu Ikeda [II10] refined the conjecture for the special orthogonal groups to give an explicit relationship between this central value of a $L$-function and the period integral and proved the first few cases. An analogous 
conjecture was formulated for unitary groups by R. Neal Harris [Har14]. The purpose of this thesis is to formulate a similar conjecture for a non-classical group known as the general spin group (GSpin), and we prove one case.

\subsection{The Gan-Gross-Prasad Conjecture}

Here we recall the original global Gross-Prasad conjecture and then discuss the IchinoIkeda refinement. We then give an analogous global conjecture for general spin groups.

\subsubsection{The Original Global Gross-Prasad Conjecture}

Let $V_{n} \subset V_{n+1}$ be quadratic spaces of respective dimensions $n$ and $n+1$. Assume $n$ is at least two and $V_{n}$ is not isomorphic to the hyperbolic plane. Consider the algebraic groups $\mathrm{SO}\left(V_{n}\right) \subset \mathrm{SO}\left(V_{n+1}\right)$ defined over a number field $F$. Let $\mathbb{A}_{F}$ be the ring of adeles defined over $F$. Denote $G_{i}:=\operatorname{SO}\left(V_{i}\right)$ and let $\pi_{n} \cong \otimes_{v} \pi_{n, v}$ and $\pi_{n+1} \cong \otimes_{v} \pi_{n+1, v}$ be irreducible tempered cuspidal automorphic representations of $G_{n}\left(\mathbb{A}_{F}\right)$ and $G_{n+1}\left(\mathbb{A}_{F}\right)$, respectively. The following is the original global conjecture proposed by Gross and Prasad.

Conjecture 1.1 (Original Global Gross-Prasad Conjecture [GP92]). Assume that for every place $v$ of $F, \operatorname{Hom}_{G_{n}\left(F_{v}\right)}\left(\pi_{n+1, v} \otimes \bar{\pi}_{n, v}, \mathbb{C}\right) \neq\{0\}$. Then there exists vectors $\phi_{i} \in \pi_{i}$ such that

$$
\int_{G_{n}(F) \backslash G_{n}\left(\mathbb{A}_{F}\right)} \phi_{n+1}\left(g_{n}\right) \overline{\phi_{n}\left(g_{n}\right)} d g_{n} \neq 0
$$

if and only if the tensor product $L$-function $L\left(1 / 2, \pi_{n+1} \times \pi_{n}\right)$ does not vanish.

Ginzburg-Jiang-Rallis have shown the forward implication, under some assumptions ([GJR05]). 


\subsubsection{The Ichino-Ikeda Refinement}

Now we discuss the refined conjecture for special orthogonal groups proposed by Ichino-Ikeda [II10]. Let $\phi_{i} \in V_{\pi_{n+1}}$ and $f_{i} \in V_{\pi_{n}}$ and consider the $G_{n}\left(\mathbb{A}_{F}\right) \times G_{n}\left(\mathbb{A}_{F}\right)$ invariant functional

$$
\mathcal{P}:\left(V_{\pi_{n+1}} \otimes \bar{V}_{\pi_{n+1}}\right) \otimes\left(V_{\pi_{n}} \otimes \bar{V}_{\pi_{n}}\right) \rightarrow \mathbb{C},
$$

defined by

$$
\mathcal{P}\left(\phi_{1}, \phi_{2}: f_{1}, f_{2}\right)=\left(\int_{G_{n}(F) \backslash G_{n}\left(\mathbb{A}_{F}\right)} \phi_{1}(g) f_{1}(g) d g\right)\left(\int_{G_{n}(F) \backslash G_{n}\left(\mathbb{A}_{F}\right)} \overline{\phi_{2}(g) f_{2}(g) d g}\right) .
$$

If $\phi_{1}=\phi_{2}$, we call it $\phi$ and similarly for $f$. Then $\mathcal{P}\left(\phi_{1}, \phi_{2}: f_{1}, f_{2}\right)=\mathcal{P}(\phi, f)$, which we call the global period integral.

Next, we develop another $G_{n}\left(\mathbb{A}_{F}\right) \times G_{n}\left(\mathbb{A}_{F}\right)$-invariant functional, but now from local integrals. For each place $v$ of $F$, let $G_{i, v}=G_{i}\left(F_{v}\right)$. Fix local pairings

$$
\mathcal{B}_{\pi_{i}, v}: \pi_{i, v} \otimes \bar{\pi}_{i, v} \rightarrow \mathbb{C}
$$

such that

$$
\mathcal{B}_{\pi_{i}}=\prod_{v} \mathcal{B}_{\pi_{i, v}} .
$$

Here the $\mathcal{B}_{\pi_{i}}$ are the Peterson pairings

$$
\begin{aligned}
\mathcal{B}_{\pi_{n}}\left(f_{1}, f_{2}\right) & =\int_{G_{n}(F) \backslash G_{n}\left(\mathbb{A}_{F}\right)} f_{1}(g) \overline{f_{2}(g)} d g \\
\mathcal{B}_{\pi_{n+1}}\left(\phi_{1}, \phi_{2}\right) & =\int_{G_{n+1}(F) \backslash G_{n+1}\left(\mathbb{A}_{F}\right)} \phi_{1}(g) \overline{\phi_{2}(g)} d g
\end{aligned}
$$

and the $d g$ are Tamagawa measures on $G_{i}\left(\mathbb{A}_{F}\right)$.

For each $v$, we consider the following $G_{n, v} \times G_{n, v}$ - invariant functional

$$
\mathcal{P}_{v}^{\natural}:\left(\pi_{n+1, v} \otimes \bar{\pi}_{n+1, v}\right) \otimes\left(\pi_{n, v} \otimes \bar{\pi}_{n, v}\right) \rightarrow \mathbb{C}
$$


defined by

$$
\begin{aligned}
& \mathcal{P}_{v}^{\natural}\left(\phi_{1, v}, \phi_{2, v} ; f_{1, v}, f_{2, v}\right)= \\
& \int_{G_{n, v}} \mathcal{B}_{\pi_{n+1, v}}\left(\pi_{n+1, v}\left(g_{n, v}\right) \phi_{1, v}, \phi_{2, v}\right) \mathcal{B}_{\pi_{n, v}}\left(\pi_{n, v}\left(g_{n, v}\right) f_{1, v}, f_{2, v}\right) d g_{n, v} .
\end{aligned}
$$

The $d g_{n, v}$ are local Haar measures chosen so that $\prod_{v} d g_{n, v}=d g_{n}$. As before, set $\mathcal{P}_{v}^{\natural}\left(\phi_{v}, \phi_{v} ; f_{v}, f_{v}\right)=\mathcal{P}_{v}^{\natural}\left(\phi_{v}, f_{v}\right)$. Ichino-Ikeda prove that if $\pi_{i, v}$ is tempered, $P_{v}^{\natural}\left(\phi_{v}, f_{v}\right)$ converges absolutely. Moreover, when $P_{v}^{\natural}\left(\phi_{v}, f_{v}\right)$ does converge, they show

$$
P_{v}^{\natural}\left(\phi_{v}, f_{v}\right)=\Delta_{G_{n+1, v}} \frac{L_{v}\left(1 / 2, \pi_{n, v} \times \pi_{n+1, v}\right)}{L_{v}\left(1, \pi_{n, v}, \mathrm{Ad}\right) L_{v}\left(1, \pi_{n+1, v}, \mathrm{Ad}\right)}
$$

where $\phi_{v}, f_{v}$ are unramified and satisfy conditions (U1)-(U6) in [II10]. Here,

$$
\Delta_{G_{n+1}, v}= \begin{cases}\zeta_{v}(2) \zeta_{v}(4) \cdots \zeta_{v}(2 l) & \text { if } \operatorname{dim} V_{n+1}=2 l+1 \\ \zeta_{v}(2) \zeta_{v}(4) \cdots \zeta_{v}(2 l-2) \cdot L_{v}(l, \mu) & \text { if } \operatorname{dim} V_{n+1}=2 l\end{cases}
$$

and $\mu=\mu_{V_{i}}$ is the Hecke character associated with the discriminant of the quadratic form associated to $V_{i}$. The $L\left(s, \pi_{n, v} \times \pi_{n+1, v}\right)$ are the tensor product $L$-functions and the $L\left(s, \pi_{i, v}, \mathrm{Ad}\right)$ are the adjoint $L$-functions. Note that $\Delta_{G_{n+1}}=L\left(M_{i}^{\vee}(1), 0\right)$, where $M_{i}^{\vee}(1)$ is the twisted dual of the motive $M_{i}$ associated to $G_{i}$ by Gross [Gro97]. We normalize by setting

$$
\mathcal{P}_{v}\left(\phi_{v}, f_{v}\right)=\Delta_{G_{n+1}}^{-1} \frac{L_{v}\left(1, \pi_{n, v}, \mathrm{Ad}\right) L_{v}\left(1, \pi_{n+1, v}, \mathrm{Ad}\right)}{L_{v}\left(1 / 2, \pi_{n, v} \times \pi_{n+1, v}\right)} \prod_{v} \mathcal{P}_{v}^{\natural}\left(\phi_{v}, f_{v}\right) .
$$

Finally, consider another $G_{n, v}\left(\mathbb{A}_{F}\right) \times G_{n, v}\left(\mathbb{A}_{F}\right)$-invariant functional,

$$
\prod_{v} \mathcal{P}_{v}:\left(V_{\pi_{n+1}} \otimes \bar{V}_{\pi_{n+1}}\right) \otimes\left(V_{\pi_{n}} \otimes \bar{V}_{\pi_{n}}\right) \rightarrow \mathbb{C}
$$

The refined global Gross-Prasad conjecture of Ichino-Ikeda gives the explicit constant of proportionality between $\mathcal{P}$ and $\prod_{v} \mathcal{P}_{v}$. 
Conjecture 1.2 (Ichino-Ikeda Refinement of the global Gross-Prasad conjecture [II10]).

$$
\mathcal{P}(\phi, f)=\frac{\Delta_{G_{n+1}}}{2^{\beta}} \frac{L\left(1 / 2, \pi_{n} \times \pi_{n+1}\right)}{L\left(1, \pi_{n}, \operatorname{Ad}\right) L\left(1, \pi_{n+1}, \operatorname{Ad}\right)} \prod_{v} \mathcal{P}_{v}\left(\phi_{v}, f_{v}\right)
$$

where $2^{\beta}$ is the product of cardinalities of the component groups attached to the $L$ packets for $\pi_{i}$.

The conjecture was proven unconditionally for $n=2$ [Wal85] and $n=3$ [Ich08]. The case for $n=4$ was handled assuming that $\pi_{5}$ is a $\theta$ lift of a representation of $\mathrm{SO}_{4}$ in [GI11].

Our goal is to provide a similar conjecture for general spin groups. For the general spin groups there exists a non-trivial center, so we adjust $\mathcal{P}$ from above as follows. Denote $\mathrm{G}_{i}=\mathrm{GSpin}\left(V_{i}\right)$ and let $\mathrm{A}_{i}$ be the identity component of the center of $\mathrm{G}_{i}$. Let $\omega_{\pi_{i}}$ be the central characters of $\pi_{i}$ restricted to $A_{i}$ and we assume $\omega_{\pi_{i}}$ are such that

$$
\omega^{2}=\omega_{\pi_{n+2}} \omega_{\pi_{n+1}} .
$$

Let $\phi_{i} \in V_{\pi_{n+1}}$ and $f_{i} \in V_{\pi_{n}}$ and consider the $G_{n}\left(\mathbb{A}_{F}\right) \times G_{n}\left(\mathbb{A}_{F}\right)$-invariant functional

$$
\mathcal{P}:\left(V_{\pi_{n+1}} \otimes \bar{V}_{\pi_{n+1}}\right) \otimes\left(V_{\pi_{n}} \otimes \bar{V}_{\pi_{n}}\right) \rightarrow \mathbb{C},
$$

defined by

$\mathcal{P}\left(\phi_{1}, \phi_{2} ; f_{1}, f_{2}\right)=\left(\int_{A_{n} \backslash G_{n}} \phi_{1}(g) f_{1}(g) \omega^{-1}(\nu(g)) d g\right)\left(\int_{A_{n} \backslash G_{n}} \overline{\phi_{2}(g) f_{2}(g) \omega^{-1}(\nu(g)) d g}\right)$.

If $\phi_{1}=\phi_{2}$, we call it $\phi$ and similarly for $f$. Then $\mathcal{P}\left(\phi_{1}, \phi_{2}: f_{1}, f_{2}\right)=\mathcal{P}(\phi, f)$, which we call the global period integral. Similarly, we set

$$
\mathcal{P}_{v}^{\natural}\left(\phi_{v}, f_{v}\right):=\int_{A_{n, v} \backslash G_{n, v}} \mathcal{B}_{\pi_{n+1, v}}\left(\pi_{n+1, v}\left(g_{n, v}\right) \phi_{v}\right) \mathcal{B}_{\pi_{n, v}}\left(\pi_{n, v}\left(g_{n, v}\right) f_{v}\right) \omega^{-1}\left(\nu\left(g_{n, v}\right)\right) d g_{n, v} .
$$


The conjecture for general spin groups is:

Conjecture 1.3 (The Gan-Gross-Prasad conjecture for general spin groups). Let $\pi_{n} \cong \otimes_{v} \pi_{n, v}$ and $\pi_{n+1} \cong \otimes_{v} \pi_{n+1, v}$ be irreducible tempered cuspidal automorphic representations of $G_{n}\left(\mathbb{A}_{F}\right)$ and $G_{n+1}\left(\mathbb{A}_{F}\right)$, respectively. Assume $\omega^{2}=\omega_{\pi_{n+1}} \omega_{\pi_{n}}$ and let $\mathcal{P}$ be as in (1.1). Then,

$$
\mathcal{P}=\frac{\Delta_{G_{n+1}}}{2^{\beta}} \frac{L\left(1 / 2, \pi_{n+1} \otimes \omega^{-1} \times \pi_{n}\right)}{L\left(1, \pi_{n+1}, \mathrm{Ad}\right) L\left(1, \pi_{n}, \mathrm{Ad}\right)} \prod_{v} \mathcal{P}_{v}\left(\phi_{v}, f_{v}\right)
$$

where

$$
\Delta_{\mathrm{G}_{i}}= \begin{cases}\zeta(2) \zeta(4) \cdots \zeta(2 m) & \text { if } \operatorname{dim} V_{i}=2 m+1, \\ \zeta(2) \zeta(4) \cdots \zeta(2 m-2) L(m, \mu) & \text { if } \operatorname{dim} V_{i}=2 m .\end{cases}
$$

Here $\mu=\mu_{V_{i}}$ is the Hecke character associated with the discriminate of the quadratic form associated to $V_{i}$, and $2^{\beta}$ is associated with the product of the cardinalities of the component groups attached to the $L$-parameters of $\pi_{n+1}$ and $\pi_{n}$. The $L\left(1, \pi_{i}, \mathrm{Ad}\right)$ are the adjoint $L$-functions defined by (2.3) and (2.4).

To discuss the term $2^{\beta}$, we first set up some notation. Let $\mathcal{L}_{F}$ be the hypothetical global Langlands group of $F$ and let $\phi_{i}: \mathcal{L}_{F} \rightarrow{ }^{L} G=\widehat{G} \rtimes W_{F}$. Set $S_{\phi_{i}}=\operatorname{Cent}\left(\operatorname{Im}\left(\phi_{i}\right), \widehat{G}\right)$. Define

$$
\mathcal{S}_{\phi_{i}}=S_{\phi_{i}} / S_{\phi_{i}}^{0} Z(\widehat{G})^{\Gamma},
$$

where, $S_{\phi_{i}}^{0}$ is the identity component of the complex reductive group $S_{\phi_{i}}, Z(\widehat{G})$ is the center of $\widehat{G}$ and $Z(\widehat{G})^{\Gamma}$ is the subgroup of invariants in $Z(\widehat{G})$ under the natural action of the Galois group $\Gamma=\operatorname{Gal}(\bar{F} / F)$.

We conjecture that 


\section{Conjecture 1.4.}

$$
2^{\beta}=4\left|\mathcal{S}_{\phi_{n}}\right|\left|\mathcal{S}_{\phi_{n+1}}\right|
$$

Remark 1.5. We work through the case where $V_{n}$ is isomorphic to a hyperbolic plane in Section 4.3. In this case, we have $\operatorname{GSpin}\left(V_{2}\right) \subseteq \operatorname{GSpin}\left(V_{3}\right)$ and $\operatorname{GSpin}_{2}$ is split. This case does not confirm Conjecture 1.3 or Conjecture 1.4.

\subsection{Outline of Dissertation}

In Chapter 2 we define general spin groups and prove two lemmas which are used throughout the dissertation. Next we review the structure theory for general spin groups and describe the root datum in the split case. We then discuss the general theory of $L$-groups and $L$-functions and describe each for GSpin groups. We conclude the chapter with a discussion on the component groups for the local Langlands parameters of GSpin in relation to Conjecture 1.4.

In Chapter 3, we begin by proving the convergence of the integral (1.2) for tempered representations at every place $v$ using results of Waldspurger [Wal85] and Silberger [Sil79]. We then compute the integral for unramified data in the split case first, then the quasi-split but non-split case. In the course of the proof, we use the doubling method of Piatetski-Shapiro and Rallis [LR05] and results of Kato-MuraseSugano [KMS03].

In Chapter 4, we prove the global Gan-Gross-Prasad conjecture proposed in Chapter 3 for $\mathrm{GSpin}_{2} \times \mathrm{GSpin}_{3}$. We discuss the Waldspurger formula ([Wal85]) before utilizing the formula in the proof of the case where $\mathrm{GSpin}_{2}$ is not split. We then work through the case when GSpin ${ }_{2}$ is split. This is an exceptional case in that it does not 
confirm Conjecture 1.3. 


\section{Chapter 2}

\section{Structure of GSpin Groups.}

In this chapter, we first define general spin groups and then prove two lemmas needed in the thesis. Next, we review the structure theory for general spin groups and describe the root datum in the split case. We then discuss the general theory of $L$-groups and $L$-functions and describe each for GSpin groups. We conclude the chapter with a discussion on the component groups for the local Langlands parameters of GSpin in relation to Conjecture 1.4.

\section{$2.1 \quad \operatorname{GSpin}(\mathrm{V})$}

We begin by defining GSpin groups and then discuss some general structure theory of GSpin groups. Good resources for the general theory of GSpin groups are [Del72].

Let $V$ be a free module over a ring $F$, equipped with a quadratic form $q$. Denote by $C(V)$ the Clifford algebra of $V$. The module $V$ is identified with a sub-module of $C(V)$. In $C(V)$, one has $v \cdot v=q(v)$. Denote by $C^{+}$the even part of $C(V)$.

Suppose that $F$ is a field of characteristic zero with a non-degenerate quadratic form $q$. The group GSpin $(V)$, also referred to as the Clifford group or as $C \operatorname{Spin}(V)$, is defined as

$$
\operatorname{GSpin}(V)=\left\{g \in C^{+}(V): g V g^{-1}=V\right\} .
$$


There is a natural homomorphism from $\operatorname{GSpin}(V)$ to $\mathrm{SO}(V)$ sending $g \in \operatorname{GSpin}(V)$ to the map $v \mapsto g v g^{-1}$. The kernel consists of scalars, giving the short exact sequence of algebraic groups

$$
1 \longrightarrow \mathrm{GL}_{1} \longrightarrow \mathrm{GSpin}(V) \longrightarrow \mathrm{SO}(V) \longrightarrow 1
$$

The spinor group $\operatorname{Spin}(V)$ is the algebraic subgroup of $\operatorname{GSpin}(V)$ which is the kernel of the similitude map

$$
\nu: \text { GSpin } \rightarrow \mathrm{GL}_{1} .
$$

Altogether this gives the commutative diagram similar to the one used by Deligne [Del72]

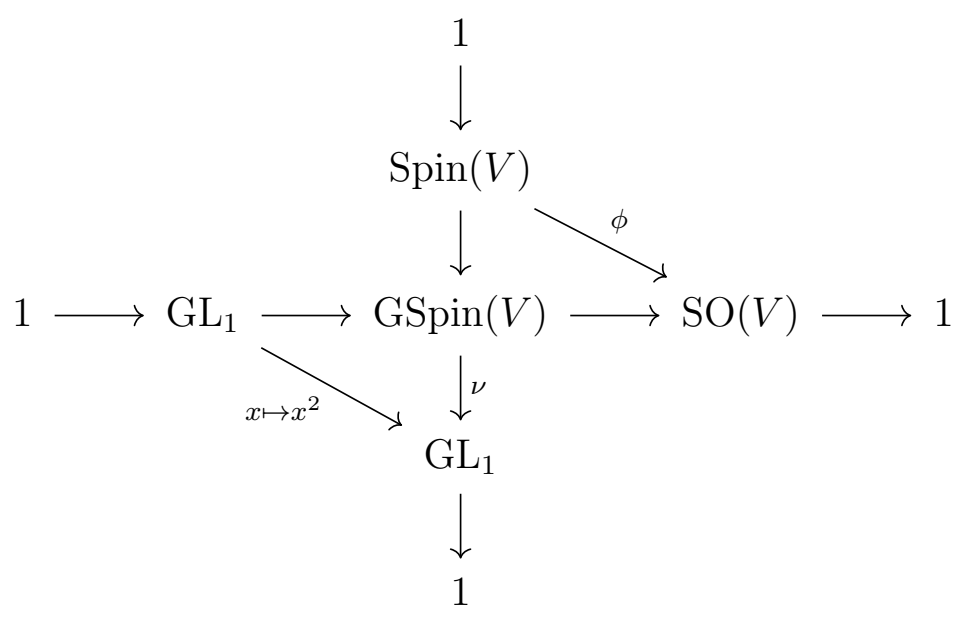

We also have the exact sequence

$$
1 \longrightarrow\{ \pm 1\} \longrightarrow \mathrm{Spin}(V) \longrightarrow \mathrm{SO}(V) \longrightarrow 1
$$

There is an identification

$$
\operatorname{GSpin}(V) \cong \frac{\mathrm{GL}_{1} \times \operatorname{Spin}(V)}{\mu_{2}}
$$

where $\mu_{2}=\operatorname{ker} \phi$ which is explicitly defined in the split case in Definition 2.4. 
Lemma 2.1. Let $\operatorname{dim} V \geq 3$, let A be the identity component of $\mathrm{GSpin}(V)$, and let $P: \operatorname{GSpin}(V) \rightarrow \mathrm{SO}(V)$. Then the kernel of $P$ is $A$, so $\mathrm{A}=\mathrm{GL}_{1}$.

Proof. Denote $Z$ to be the center of $\operatorname{GSpin}(V)$ and denote $Z_{\mathrm{SO}}$ to be the center of $\mathrm{SO}(V)$. Let $a \in Z$. From $(2.1)$ we have

$$
1 \longrightarrow \mathrm{GL}_{1} \longrightarrow \mathrm{GSpin}(V) \stackrel{P}{\longrightarrow} \mathrm{SO}(V) \longrightarrow 1
$$

There is a homomorphism sending $a$ to $\bar{a}$ in $Z_{\mathrm{SO}}$. Recall that $Z_{\mathrm{SO}}=\{ \pm 1\}$. The center of $\operatorname{GSpin}(V)$ must be in $P^{-1}(\{ \pm 1\})$. Note that $P^{-1}(\{1\})=\operatorname{ker} P$ and $\operatorname{ker} P=\mathrm{GL}_{1}$. Let $A$ be the identity component of $Z$. Since $\{ \pm 1\}$ is disconnected, and $\mathrm{GL}_{1}$ is connected,

$$
A \subseteq P^{-1}(\{1\})=\mathrm{GL}_{1} \subseteq Z
$$

implies $A=\mathrm{GL}_{1}$.

Lemma 2.2. Let $V_{n}, V_{n+1}$ be quadratic spaces over $F$ such that $V_{n} \subseteq V_{n+1}$. One has the commutative diagram

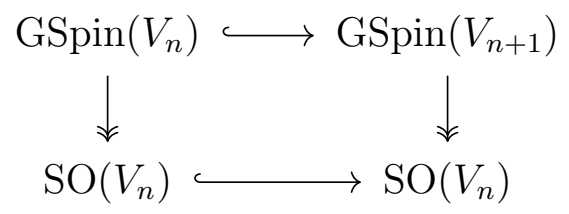

Proof. Let $V_{n} \subseteq V_{n+1}$. Then there exists a natural injection from $\mathrm{SO}\left(V_{n}\right)$ to $\mathrm{SO}\left(V_{n+1}\right)$.

From (2.1) we have the exact sequence

$$
1 \longrightarrow \mathrm{GL}_{1} \longrightarrow \operatorname{GSpin}\left(V_{n+1}\right) \longrightarrow \mathrm{SO}\left(V_{n+1}\right) \stackrel{P}{\longrightarrow} 1
$$

which gives the exact sequence

$$
1 \longrightarrow \mathrm{GL}_{1} \longrightarrow P^{-1}\left(\mathrm{SO}\left(V_{n}\right)\right) \longrightarrow \mathrm{SO}\left(V_{n}\right) \longrightarrow 1
$$


Because $P^{-1}\left(\mathrm{SO}\left(V_{n}\right)\right)$ is a non-trivial extension of $\mathrm{SO}\left(V_{n}\right)$ by $\mathrm{GL}_{1}, P^{-1}\left(\mathrm{SO}\left(V_{n}\right)\right)=$ $\operatorname{GSpin}\left(V_{n}\right)$. Thus, $\operatorname{GSpin}\left(V_{n}\right) \subseteq \operatorname{GSpin}\left(V_{n+1}\right)$.

Next, we discuss some general structure theory of $\operatorname{GSpin}(V)$ which will be utilized in the proof of Proposition 3.3.

Let $F$ be a local field with residual characteristic not 2 . Let $V$ be a non-degenerate quadratic space over $F$. Denote the anisotropic kernel of $V$ by $V^{a n}$ and denote the dimension of $V^{a n}$ by $d$. Then there is a decomposition

$$
V=X \oplus V^{a n} \oplus Y
$$

where $X$ and $Y$ are totally isotropic spaces. By definition, the Witt rank $r$ equals the dimension of $X$ or $Y$. By fixing a basis for $X$, we obtain a minimal parabolic subgroup $P_{\min }=M_{\min } N_{\min }$ of GSpin $(V)$. The Levi factor $M_{\min }$ is isomorphic to the direct product of $\left(F^{\times}\right)^{r} \times \mathrm{GSpin}_{V^{a n}}$. The split component $A_{\min }$ of $M_{\min }$ is isomorphic to $\left(F^{\times}\right)^{r}$ and the Weyl group $W\left(\operatorname{GSpin}(V), A_{\min }\right)$ is of type $B$ if $d$ is odd and is of type $D$ if $d$ is even. Denote an element $A_{\min }$ by $x=\left(x_{1}, x_{2}, x_{3}, \ldots, x_{r}\right)$. The simple roots of $\left(P_{\min }, A_{\min }\right)$ are given by

$$
\alpha_{1}(x)=x_{1} x_{2}^{-1}, \ldots, \alpha_{r-1}(x)=x_{r-1} x_{r}^{-1}
$$

and

$$
\alpha_{r}= \begin{cases}x_{r} & \text { if } d \text { is odd } \\ x_{r-1} x_{r} & \text { if } d \text { is even. }\end{cases}
$$

Let $T$ be a maximal torus such that $T \subset M_{\text {min }}$. We view the $\alpha_{i}$ as elements of $\operatorname{Hom}\left(T, \mathrm{GL}_{1}\right)$ via the natural projection $M_{\min } \rightarrow T$. This allows us to view these 
roots as a character of $M_{\min }$. Let $\delta_{P_{\min }}(x)$ be the modulus character of $P_{\min }$. Then

$$
\delta_{P_{\min }}(x)=\prod_{i=1}^{r}\left|x_{i}\right|^{d+2 r-2 i}
$$

\subsection{Root Datum for split GSpin}

In this section, we describe split $\mathrm{GSpin}_{n}$ in terms of its root datum.

Proposition 2.3. The root datum of $\mathrm{GSpin}_{n}, n=2 m+1$ or $2 m$, can be described as the following. Let

$$
\begin{aligned}
X & =\mathbb{Z} e_{o} \oplus \mathbb{Z} e_{1} \oplus \cdots \oplus \mathbb{Z} e_{m}, \\
X^{\vee} & =\mathbb{Z} e_{o}^{*} \oplus \mathbb{Z} e_{1}^{*} \oplus \cdots \oplus \mathbb{Z} e_{m}^{*},
\end{aligned}
$$

and let

$$
\langle,\rangle: X \times X^{\vee} \rightarrow \mathbb{Z}
$$

be the standard pairing. The root datum for $\mathrm{GSpin}_{n}$ is $\left(X, R, X^{\vee}, R^{\vee}\right)$ with $R$ and and $R^{\vee}$ generated by the simple roots and coroots given by, respectively,

$$
\begin{aligned}
\Delta & =\left\{\alpha_{1}=e_{1}-e_{2}, \alpha_{2}=e_{2}-e_{3}, \ldots, \alpha_{m-1}=e_{m-1}-e_{m}, \alpha_{m}=e_{m}\right\}, \\
& \Delta^{\vee}=\left\{\alpha_{1}^{\vee}=e_{1}^{*}-e_{2}^{*}, \alpha_{2}^{\vee}=e_{2}^{*}-e_{3}^{*}, \ldots, \alpha_{m-1}^{\vee}=e_{m-1}^{*}-e_{m}^{*}, \alpha_{m}^{\vee}=2 e_{m}^{*}-e_{0}^{*}\right\}, \\
\text { if } n=2 m & +1 \text { and by } \\
\Delta & =\left\{\alpha_{1}=e_{1}-e_{2}, \alpha_{2}=e_{2}-e_{3}, \ldots, \alpha_{m-1}=e_{m-1}-e_{m}, \alpha_{m}=e_{m-1}+e_{m}\right\}, \\
\Delta^{\vee} & =\left\{\alpha_{1}^{\vee}=e_{1}^{*}-e_{2}^{*}, \ldots, \alpha_{m-1}^{\vee}=e_{m-1}^{*}-e_{m}^{*}, \alpha_{m}^{\vee}=e_{m-1}^{*}+e_{m}^{*}-e_{0}^{*}\right\}, \\
\text { if } n=2 m &
\end{aligned}
$$

Proof. See [Asg02, Section 2]. 
The root datum listed in Proposition 2.3 corresponds with the root datum listed in Section 2.1. For example, $\alpha_{1}(x)=\left(e_{1}-e_{2}\right)(x)=x_{1} x_{2}^{-1}$. The root datum of Proposition 2.3 determines the split group $\mathrm{GSpin}_{n}$ uniquely, equipped with a Borel subgroup containing a maximal torus $T$. Note that $\alpha_{i}: T \rightarrow G L_{1}$ and $\alpha_{i}^{\vee}: G L_{1} \rightarrow T$ is such that $\alpha_{i} \circ \alpha_{j}^{\vee}(x)=x^{\left\langle\alpha_{i}, \alpha_{j}^{\vee}\right\rangle}$.

When $n=2 m+1$, GSpin $n$ has a Dynkin diagram of type $B_{m}$ :

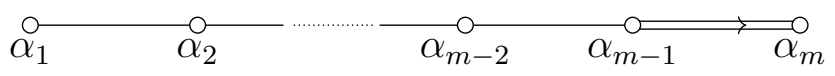

When $n=2 m$, GSpin $_{n}$ has a Dynkin diagram of type $D_{m}$ :

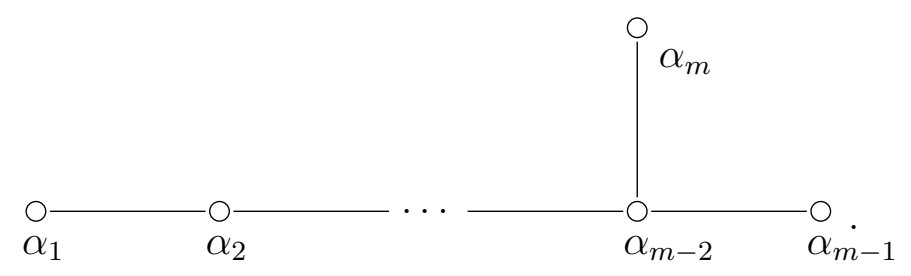

Now that the root structure for GSpin has been described explicitly, we can define split GSpin as follows:

Proposition 2.4. For $n \geq 3$,

$$
\mathrm{GSpin}_{n} \cong \frac{\mathrm{GL}_{1} \times \operatorname{Spin}_{n}}{\{(1,1),(-1, c)\}}
$$

where $c=\alpha_{m}^{\vee}(-1)$ if $n=2 m+1$ and $c=\alpha_{m-1}^{\vee}(-1) \alpha_{m}^{\vee}(-1)$ if $n=2 m$.

Proof. See [Asg02, Section 2]

We define $\operatorname{GSpin}_{0}=\mathrm{GSpin}_{1}=\mathrm{GL}_{1}$. See the $n=2$ case for a definition of GSpin ${ }_{2}$.

Proposition 2.5. The center of $\mathrm{GSpin}_{n}$ is given by

$$
Z\left(\mathrm{G}_{n}\right)= \begin{cases}\mathrm{A}_{0} & \text { if } n=2 m+1, \\ \mathrm{~A}_{0} \cup\left(\zeta_{0} A_{0}\right) & \text { if } n=2 m\end{cases}
$$

where $\mathrm{A}_{0}=\left\{e_{0}^{*}(\lambda): \lambda \in \mathrm{GL}_{1}\right\}$ and $\zeta_{0}=e_{1}^{*}(-1) e_{2}^{*}(-1) \cdots e_{m}^{*}(-1)$.

Proof. See [AS06, Prop. 2.3]. 


\section{$2.3 \quad L$-groups}

In this section, we first discuss the general theory of $L$-groups, and then describe the $L$-groups of GSpin. For the concepts reviewed in this section, one may consult [Roh94] or [CKM04, Ch. 3].

Let $k$ be a finite field of characteristic $p$ and cardinality $q$. We denote $\bar{k}$ as the algebraic closure of $k$. The Weil group of $k$, denoted $W_{k}$, is the infinite cyclic subgroup of $\operatorname{Gal}(\bar{k} / k)$ generated by the inverse of the Frobenius automorphism.

Now consider a nonarchimedean local field $F$ with residue field $k$. Denote the separable algebraic closure of $F$ as $\bar{F}$ and $F_{u n r}$ for the maximal unramified extension of $F$. The inertia subgroup of $\operatorname{Gal}(\bar{F} / F)$ is defined to be $I_{F}=\operatorname{Gal}\left(\bar{F} / F_{\text {unr }}\right)$. Identifying $\bar{k}$ with the residue field of $\bar{F}$ (or of $F_{u n r}$ ), we have an exact sequence

$$
1 \longrightarrow I_{F} \longrightarrow \operatorname{Gal}(\bar{F} / F) \stackrel{\pi}{\longrightarrow} \operatorname{Gal}(\bar{k} / k) \longrightarrow 1
$$

where $\pi$ is the decomposition map. Define $W_{F}$ to be the inverse image of $W_{k}$ under $\pi:$

$$
W_{F}=\pi^{-1}\left(W_{k}\right)
$$

Then $W_{F}$ fits into the exact sequence

$$
1 \longrightarrow I_{F} \longrightarrow W_{F} \longrightarrow W_{k} \longrightarrow 1
$$

Denote the inverse of the Frobenius automorphism as Frob, and one has the exact sequence

$$
1 \longrightarrow I_{F} \longrightarrow W_{F} \longrightarrow\langle F r o b\rangle \longrightarrow 1 \text {. }
$$

Since $\langle F r o b\rangle \subseteq \operatorname{Gal}(\bar{k} / k), W_{F}$ is a subgroup of $\operatorname{Gal}(\bar{F} / F)$. By a representation of $W_{F}$ we mean a continuous homomorphism $W_{F} \rightarrow \mathrm{GL}(V)$, where $V$ is a finite-dimensional 
complex vector space. A representation $\sigma$ of $W_{F}$, is unramified if $\sigma$ restricted to $I_{F}$ is trivial, and ramified otherwise.

Let $G$ be a connected, reductive group over $F$. Let $\widehat{G}$ denote the complex dual of $G$. The $L$-group of $G$ is denoted ${ }^{L} G$ and ${ }^{L} G=\widehat{G} \rtimes W_{F}$ or $\widehat{G} \rtimes G a l(\bar{F} / F)$. The following is a list of groups and the respective dual groups.

\begin{tabular}{ll||ll}
\hline $\mathrm{G}$ & $\widehat{G}$ & $\mathrm{G}$ & $\widehat{G}$ \\
\hline $\mathrm{GL}_{m}$ & $\mathrm{GL}_{m}(\mathbb{C})$ & $\mathrm{SL}_{m}$ & $\mathrm{PGL}_{m}(\mathbb{C})$ \\
$\mathrm{SO}_{2 m+1}$ & $\mathrm{Sp}_{2 m}(\mathbb{C})$ & $\mathrm{GSp}_{2 m}$ & $\mathrm{GSpin}_{2 m+1}(\mathbb{C})$ \\
$\mathrm{SO}_{2 m}$ & $\mathrm{SO}_{2 m}(\mathbb{C})$ & $\mathrm{GSO}_{2 m}$ & $\mathrm{GSpin}_{2 m}(\mathbb{C})$ \\
\hline
\end{tabular}

Hence, the $L$-groups of quasi-split GSpin groups are

$$
{ }^{L} \operatorname{GSpin}_{n}=\mathrm{GSp}_{2 m}(\mathbb{C}) \rtimes W_{F}
$$

if $n=2 m+1$, and

$$
{ }^{L} \mathrm{GSpin}_{n}=\mathrm{GSO}_{2 m}(\mathbb{C}) \rtimes W_{F}
$$

if $n=2 m$.

When $G$ is split, the semi-direct product is a direct product. If $\mathrm{GSpin}_{n}$ is not quasi-split, then there exists a quasi-split GSpin* ${ }_{n}^{*}$ so that GSpin is an inner form of $\operatorname{GSpin}_{n}^{*}$. Then

$$
{ }^{L} \operatorname{GSpin}_{n}={ }^{L} \operatorname{GSpin}_{n}{ }^{*} .
$$

When $F$ is a global field, $G$ is quasi-split and unramified, i.e. split over an unramified extension of $F_{v}$ for almost all places $v$ of $F$ ([Sha10, pg. 7]). 


\section{$2.4 \quad L$-functions for GSpin}

First we develop the local $L$-functions for split GSpin, and then quasi-split but nonsplit. We conclude the chapter by defining the global (or automorphic) $L$-functions for GSpin .

\subsection{1 $L$-functions}

In the general setting, let $G$ be a connected reductive group over a number field $F$. Let $\pi$ be an automorphic representation. For almost all $v$, the local representation $\pi_{v}$ is an unramified representation of $G\left(F_{v}\right)$. Here $v$ is a non-archimedean place. Let $G_{v}=G\left(F_{v}\right)$ in this section. To develop $L$-functions, we consider representations of the Weil-Deligne group $W D_{F_{v}}$ which is defined to be

$$
W D_{F_{v}}=W_{F_{v}} \times S L_{2}(\mathbb{C})
$$

The Local Langlands Conjecture asserts that there is a surjective, finite-to-one map from the set $\operatorname{Irr}\left(G_{v}\right)$ of isomorphism classes of irreducible smooth complex representations of $G\left(F_{v}\right)$ to the set of admissible homomorphisms $\phi: W D_{F_{v}} \rightarrow{ }^{L} G_{v}$.

Now let $G_{v}=\operatorname{GSpin}_{2 m+1}\left(F_{v}\right)$. Assume that $G_{v}$ is split. Then $\widehat{G}_{v}=\operatorname{GSp}_{2 m}(\mathbb{C})$. The Local Langlands Conjecture gives

$$
\operatorname{Irr}\left(G_{v}\right) \rightarrow\left\{\phi: W D_{F_{v}} \rightarrow \operatorname{GSp}_{2 m}(\mathbb{C})\right\}
$$

sending $\pi_{n+1, v}$ to $\phi_{\pi_{n+1, v}}$.

Let $\pi_{n+1, v}$ be unramified. Conjecturally, $\phi_{\pi_{n+1, v}}$ is trivial on $\mathrm{SL}_{2}(\mathbb{C})$ and on $I_{F_{v}}$. Then we have that

$$
\phi_{\pi_{n+1, v}}: I_{F_{v}} \backslash W_{F_{v}} \rightarrow \operatorname{GSp}_{2 m}(\mathbb{C})
$$


From the exact sequence $(2.2), I_{F_{v}} \backslash W_{F_{v}} \cong\langle F r o b\rangle \cong \mathbb{Z}$. Denote $\varpi_{F}$ as the uniformizer of $F_{v}$. Since $\pi_{n+1, v}$ is unramified, it is given by an unramified character $\Xi$ of the maximal torus $T\left(F_{v}\right)$. This means that there are unramified characters $\Xi_{0}, \Xi_{1}, \ldots, \Xi_{m}$ of $F_{v}^{\times}$such that we have the following ([AS06, proof of Thm 1.1]):

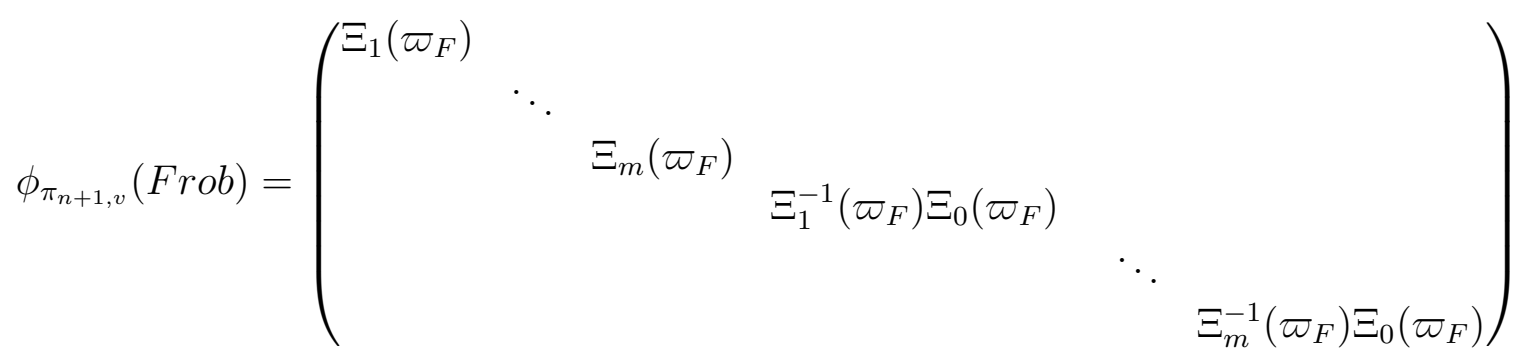

which is a subset of $\mathrm{GSp}_{2 m}(\mathbb{C})$. Let $q$ be the cardinality of the residue field. The standard local $L$-function for $\pi_{n+1, v}$ is

$$
\begin{aligned}
L\left(s, \pi_{n+1, v}\right)=L\left(s, \phi_{\pi_{n+1, v}}\right) & =\operatorname{det}\left(I-q^{-s} \phi_{\pi_{v}}(F r o b)\right)^{-1} \\
& =\prod_{i=1}^{m} \frac{1}{1-q^{-s} \Xi_{i}\left(\varpi_{F}\right)} \frac{1}{1-q^{-s} \Xi_{i}^{-1} \Xi_{0}\left(\varpi_{F}\right)} \\
& =\prod_{i=1}^{m} L\left(s, \Xi_{i}\right) L\left(s, \Xi_{i}^{-1} \Xi_{0}\right) .
\end{aligned}
$$

Let $n=2 m$ be even and assume GSpin $n$ is split. Then similarly, if we assume $\pi_{n, v}$ is unramified, it is given by an unramified character $\xi$ of the maximal torus $T\left(F_{v}\right)$. This means that there are unramified characters $\xi_{0}, \xi_{1}, \ldots, \xi_{m}$ of $F_{v}^{\times}$such that we have the following

$$
\phi_{\pi_{n, v}}(F r o b)=\left(\begin{array}{llllll}
\xi_{1}\left(\varpi_{F}\right) & & & & & \\
& \ddots & & & & \\
& & \xi_{m}\left(\varpi_{F}\right) & & & \\
& & & \xi_{1}^{-1}\left(\varpi_{F}\right) \xi_{0}\left(\varpi_{F}\right) & & \\
& & & & \ddots & \\
& & & & & \xi_{m}^{-1}\left(\varpi_{F}\right) \xi_{0}\left(\varpi_{F}\right)
\end{array}\right)
$$

which is a subset of $\mathrm{GSO}_{2 m}(\mathbb{C})$. The standard local $L$-function for $\pi_{n, v}$ is

$$
L\left(s, \pi_{n, v}\right)=L\left(s, \phi_{\pi_{n, v}}\right)=\operatorname{det}\left(I-q^{-s} \phi_{\pi_{v}}(F r o b)\right)^{-1}
$$




$$
\begin{aligned}
& =\prod_{i=1}^{m} \frac{1}{1-q^{-s} \xi_{i}\left(\varpi_{F}\right)} \frac{1}{1-q^{-s} \xi_{i}^{-1} \xi_{0}\left(\varpi_{F}\right)} \\
& =\prod_{i=1}^{m} L\left(s, \xi_{i}\right) L\left(s, \xi_{i}^{-1} \xi_{0}\right) .
\end{aligned}
$$

Let $n=2 m$, and suppose $\pi_{v}$ is unramified and assume GSpin ${ }_{n}$ is quasi-split, but not split. There is a one-to-one correspondence between $\pi_{v}$ and the Satake parameters for $\mathrm{GSpin}_{n}$ given by [AS14, (3.32)]

$$
\left(\begin{array}{ccccc}
\chi_{1}\left(\varpi_{F}\right) & & & & \\
& \ddots & & & \\
& \chi_{m}\left(\varpi_{F}\right) \mu & & & \\
& & \chi_{1}^{-1}\left(\varpi_{F}\right) \chi_{0}\left(\varpi_{F}\right) & & \\
& & & \ddots & \\
& & & & \chi_{m}^{-1}\left(\varpi_{F}\right) \chi_{0}\left(\varpi_{F}\right)
\end{array}\right)
$$

where $\mu=\mu_{V_{n}}$ is the Hecke character associated with the discriminate of the quadratic form associated to $V_{n}$. Then,

$$
\begin{aligned}
L\left(s, \pi_{v}\right) & =L\left(s, \phi_{\pi_{v}}\right)=\operatorname{det}\left(I-q^{-s} \phi_{\pi_{v}}(F r o b)\right)^{-1} \\
& =\frac{1}{1-q^{-s} \chi_{m}\left(\varpi_{F}\right) \mu} \frac{1}{1-q^{-s} \chi_{m}\left(\varpi_{F}\right)} \prod_{i=1}^{m-1} \frac{1}{1-q^{-s} \chi_{i}\left(\varpi_{F}\right)} \frac{1}{1-q^{-s} \chi_{i}^{-1} \chi_{0}\left(\varpi_{F}\right)} \\
& =L\left(s, \chi_{m} \mu\right) L\left(s, \chi_{m}^{-1} \chi_{0}\right) \prod_{i=1}^{m-1} L\left(s, \chi_{i}\right) L\left(s, \chi_{i}^{-1} \chi_{0}\right) .
\end{aligned}
$$

\subsubsection{Tensor product L-functions}

Assume that GSpin ${ }_{n}$ and $\operatorname{GSpin}_{n+1}$ are split and assume that $\pi_{n+1}$ and $\pi_{n}$ are unramified. Using the standard $L$-functions, the local tensor product $L$-functions, are

$$
\begin{array}{rl}
L\left(s, \pi_{n+1} \times \pi_{n}\right)=\prod_{1 \leq i, j \leq m} & L\left(s, \Xi_{i} \xi_{j}\right) L\left(s, \Xi^{-1} \xi_{j} \Xi_{0}\right) \\
& \times L\left(s, \Xi_{i} \xi_{j}^{-1} \xi_{0}\right) L\left(\Xi_{i}^{-1} \xi_{j}^{-1} \Xi_{0} \xi_{0}\right) .
\end{array}
$$




\subsubsection{Adjoint $L$-functions}

Here we define what we mean by $L(s, \pi, \mathrm{Ad})$ in Conjecture 1.3. Let $G=\mathrm{GSpin}_{2 n+1}$ then $\widehat{G}=\operatorname{GSp}_{2 n}(\mathbb{C})$ and $\widehat{\mathrm{SO}}_{2 n+1}=\operatorname{Sp}_{2 n}(\mathbb{C})$. Let $\pi_{v}$ be an irreducible admissible representation of $G_{v}$. Then one has the following

$$
W D_{F_{v}} \stackrel{\phi_{\pi_{v}}}{\longrightarrow} \operatorname{GSp}_{2 n}(\mathbb{C}) \stackrel{\operatorname{Ad}_{\mathfrak{g} \mathfrak{p} \mathfrak{p}}}{\longrightarrow} \operatorname{GL}(\operatorname{Lie}(\widehat{G}))
$$

Note that $\operatorname{Lie}(\widehat{G})=\mathfrak{g s p}_{2 n}(\mathbb{C})=\operatorname{Lie}\left(\operatorname{Sp}_{2 n}\right)(\mathbb{C}) \oplus \mathbb{C}$. Hence, $\operatorname{Ad}_{\mathfrak{g s p}}=\operatorname{Ad}_{\mathfrak{s p}} \oplus \mathbb{1}$. Then,

$$
L\left(s, \pi_{v}, \operatorname{Ad}_{\mathfrak{g} \mathfrak{s p}}\right)=L\left(s, \pi_{v}, \operatorname{Ad}_{\mathfrak{s p}}\right) \zeta_{v}(s)
$$

and we have

$$
\begin{aligned}
L\left(s, \pi, \operatorname{Ad}_{\mathfrak{g} \mathfrak{s p}}\right) & :=\prod_{v} L\left(s, \pi_{v}, \operatorname{Ad}_{\mathfrak{g} \mathfrak{s p}}\right) \\
& =\prod_{v} L\left(s, \pi_{v}, \operatorname{Ad}_{\mathfrak{s p}}\right) \zeta_{v}(s) \\
& =L\left(s, \pi, \operatorname{Ad}_{\mathfrak{s p}}\right) \zeta(s) .
\end{aligned}
$$

We define

$$
L(s, \pi, \mathrm{Ad}):=L\left(s, \pi, \operatorname{Ad}_{\mathfrak{s p}}\right)
$$

Similarly, if $G=\mathrm{GSpin}_{2 n}$, in the split case we define

$$
L(s, \pi, \operatorname{Ad})=L\left(s, \pi, \operatorname{Ad}_{\mathfrak{s o}}\right)
$$

\subsection{Component Groups for the local Langlands pa- rameters}

This goal of this section is to discuss the component groups for the local Langlands

parameters in relation to Conjecture 1.4. We restate the notation from Chapter 1 for 
the sake of the reader. Let $\mathcal{L}_{F}$ be the hypothetical global Langlands group of $F$ and let $\phi_{i}: \mathcal{L}_{F} \rightarrow{ }^{L} G=\widehat{G} \rtimes W_{F}$. Set $S_{\phi_{i}}=\operatorname{Cent}\left(\operatorname{Im}\left(\phi_{i}\right), \widehat{G}\right)$. Define

$$
\mathcal{S}_{\phi_{i}}=S_{\phi_{i}} / S_{\phi_{i}}^{0} Z(\widehat{G})^{\Gamma}
$$

where, $S_{\phi_{i}}^{0}$ is the identity component of the complex reductive group $S_{\phi_{i}}, Z(\widehat{G})$ is the center of $\widehat{G}$ and $Z(\widehat{G})^{\Gamma}$ is the subgroup of invariants in $Z(\widehat{G})$ under the natural action of the Galois group $\Gamma=\operatorname{Gal}(\bar{F} / F)$.

Recall, we conjecture (Conjecture 1.4)

$$
2^{\beta}=4\left|\mathcal{S}_{\phi_{n}}\right|\left|\mathcal{S}_{\phi_{n+1}}\right|
$$

We justify this conjecture with the following. Let $\pi$ be a cuspidal representation of split GSpin $2 n+1$. The global Langlands conjecture states that

$$
\phi_{\pi}: \mathcal{L}_{F} \rightarrow{ }^{L} \mathrm{GSpin}_{2 n+1}=\mathrm{GSp}_{2 n}(\mathbb{C}) \times W_{F}
$$

and $\operatorname{Im}\left(\phi_{\pi}\right)$ is not in a proper Levi of $\operatorname{GSp}_{2 n}(\mathbb{C})$. Then

$$
\phi_{\pi}: \mathcal{L}_{F} \rightarrow \mathrm{GSp}_{2 n}(\mathbb{C})=\operatorname{GSp}(W)
$$

where $(W,\langle\cdot, \cdot\rangle)$ is a symplectic space over $\mathbb{C}$ of $\operatorname{dim}_{\mathbb{C}} W=2 n$.

For $l \in \mathcal{L}_{F}$,

$$
\left\langle\phi_{\pi}(l) w, \phi_{\pi}(l) w^{\prime}\right\rangle=\nu(l)\left\langle w, w^{\prime}\right\rangle
$$

where $\nu(l) \in \mathbb{C}^{\times}$is the similitude character discussed in Chapter 2 .

This implies that $\phi_{\pi}$ is a representation of $\mathcal{L}_{F}$ acting on $W$. Let $W_{0} \subseteq W$ be an irreducible subspace of $\phi_{\pi}$. Define

$$
W_{0}^{\perp}=\left\{w \in W:\left\langle w, w_{0}\right\rangle=0 \text { for all } w_{0} \in W_{0}\right\}
$$


Note that $W_{0}^{\perp}$ is a subrepresentation of $\phi_{\pi}$. To see this, let $l \in \mathcal{L}_{F}$ and $w \in W_{0}^{\perp}$. We want to show that $\phi_{\pi}(l) w \in W_{0}^{\perp}$. Consider

$$
\left\langle\phi_{\pi}(l) w, w_{0}\right\rangle=\left\langle\phi_{\pi}(l) w, \phi_{\pi}(l) \phi_{\pi}(l)^{-1} w_{0}\right\rangle=\nu(l)\left\langle w, \phi_{\pi}(l)^{-1} w_{0}\right\rangle=0
$$

Assume that $W_{0} \cap W_{0}^{\perp}=0$ or $W_{0} \cap W_{0}^{\perp}=W_{0}$. If $W_{0} \cap W_{0}^{\perp}=0$ then this implies that $W=W_{0} \oplus W_{0}^{\perp}$. If $W_{0} \cap W_{0}^{\perp}=W_{0}$ then $W_{0} \subseteq W_{0}^{\perp}$. But if $w_{0}, w_{0}^{\prime} \in W_{0}$ and $\left\langle w_{0}, w_{0}^{\prime}\right\rangle=0$, then $W_{0}$ is totally isotropic. Hence $W_{0}$ is not a subset of $W_{0}^{\perp}$.

Note that $\operatorname{Im}\left(\phi_{\pi}\right)$ fixes $W_{0}$ setwise. $\operatorname{Im}\left(\phi_{\pi}\right)$ stabilizes the flag $0 \subseteq W_{0} \subseteq W$ which implies $\operatorname{Im}\left(\phi_{\pi}\right)$ is in the parabolic subgroup $P\left(W_{0}\right) \subseteq \operatorname{GSp}(W)$. Then $\operatorname{Im}\left(\phi_{\pi}\right)$ is in the Levi of $P\left(W_{0}\right)$, which is a contradiction.

Hence, $W=W_{0} \oplus W_{0}^{\perp}$ which means

$$
W=W_{1} \oplus W_{2} \oplus \cdots \oplus W_{k} .
$$

where each $\left(W_{i},\langle\cdot, \cdot\rangle,\right)$ is a smaller symplectic space. Then

$$
\operatorname{Im}\left(\phi_{\pi}\right) \subseteq\left(\operatorname{GSp}\left(W_{1}\right) \times \cdots \times \operatorname{GSp}\left(W_{k}\right)\right) \cap \operatorname{GSp}(W)
$$

and there are similitude characters $\nu_{i}$ corresponding to each of the $\operatorname{GSp}\left(W_{i}\right)$. This forces

$\operatorname{Im}\left(\phi_{\pi}\right) \subseteq\left\{\left(g_{1}, g_{2}, \cdots, g_{k}\right) \in \operatorname{GSp}\left(W_{1}\right) \times \cdots \times \operatorname{GSp}\left(W_{k}\right): \nu\left(g_{1}\right)=\nu\left(g_{2}\right)=\cdots=\nu\left(g_{k}\right)\right\}$

Then,

$$
\operatorname{Cent}\left(\operatorname{Im}\left(\phi_{\pi}\right)=\left\{\left(a_{1}, a_{2},, \ldots, a_{k}\right): a_{1}^{2}=a_{2}^{2}=\cdots a_{k}^{2}\right\}=\{( \pm a, \pm a, \pm a, \cdots, \pm a)\}\right.
$$

and so $\operatorname{Cent}\left(\operatorname{Im}\left(\left.\phi_{\pi}\right|_{W_{1}}\right)\right)=\mathbb{C}^{\times}=Z\left(\operatorname{GSp}\left(W_{1}\right)\right)$. Then

$S_{\phi} / Z(\operatorname{GSp}(W))=\{( \pm 1, \pm 1, \cdots, \pm 1)\}=(\mathbb{Z} / 2 \mathbb{Z})^{k}$. Finally

$$
\mathcal{S}_{\phi}=(\mathbb{Z} / 2 \mathbb{Z})^{k-1}
$$




\section{Chapter 3}

\section{Local Integrals of Matrix Coefficients}

In this chapter, we begin by proving the integral 1.2 converges at every place $v$ assuming the representations are tempered. The proof uses results of Waldspurger [Wal03] and Silberger [Sil79]. We then compute the integral for unramified data in the split case followed by the quasi-split but non-split case. In the course of the proof, we use the doubling method of Piatetski-Shapiro and Rallis [LR05] and results of Kato-Murase-Sugano [KMS03].

We utilize the notation from chapter one. Let $V_{n+1} \subseteq V_{n+2}$ be quadratic spaces of dimension $n+1$ and $n+2$, respectively. Let $F$ be a number field with adele ring $\mathbb{A}_{F}$ and let $\mathrm{G}_{i}=\operatorname{GSpin}\left(V_{i}\right)$. From Lemma 2.2, we consider the algebraic groups $\operatorname{GSpin}\left(V_{n+1}\right) \subseteq \operatorname{GSpin}\left(V_{n+2}\right)$ defined over $F$. Let $\pi_{n+1} \cong \otimes_{v} \pi_{n+1, v}$ and $\pi_{n+2} \cong$ $\otimes_{v} \pi_{n+2, v}$ be irreducible tempered cuspidal automorphic representations of $\mathrm{G}_{n+1}$ and $\mathrm{G}_{n+2}$, respectively. Let $S$ denote a sufficiently large finite set of bad places, including all even and archimedean places. Assume the following holds for all $v \notin S$ :

1. $\mathrm{G}_{i, v}$ is unramified over $F_{v}$

2. $K_{i, v}$ is a hyperspecial maximal compact subgroup of $\mathrm{G}_{i, v}$ 
3. $K_{i, v} \subset K_{i+1, v}$

4. $\pi_{i, v}$ is an unramified representation of $\mathrm{G}_{i, v}$

5. The vectors $f_{i, v} \in \pi_{i, v}$ are fixed by $K_{i, v}$ and $\left\|f_{i, v}\right\|=1$

6. $\int_{K_{i, v}} d g_{i, v}=1$.

For $v$ not in $S$, we still fix a maximal compact subgroup $K_{i}$ of $G_{i}$. For the remainder of this chapter, we omit the $v$ from the notation, though everything is local.

Put

$$
L_{\pi_{i+1}, \pi_{i}}(s):=\frac{L\left(s, \pi_{i+1} \otimes \omega^{-1} \times \pi_{i}\right)}{L\left(s+1 / 2, \pi_{i+1}, \mathrm{Ad}\right) L\left(s+1 / 2, \pi_{i}, \mathrm{Ad}\right)} .
$$

Consider the matrix coefficients

$$
\Phi_{\phi_{i}, \phi_{i}^{\prime}}\left(g_{i}\right):=\mathcal{B}_{\pi_{i}}\left(\pi_{i}(g) \phi_{i}, \phi_{i}^{\prime}\right), \quad g_{i} \in \mathrm{G}_{i}
$$

for $K_{i}$ - finite vectors $\phi_{i}, \phi_{i}^{\prime} \in \pi_{i}$. When $\phi_{i}=\phi_{i}^{\prime}$ we refer only to $\Phi_{\phi_{i}}$. Denote $\mathrm{A}_{i}$ as the identity component of the center of $\mathrm{G}_{i}$ and let $\omega_{\pi_{i}}$ be the central characters of $\pi_{i}$ restricted to $\mathrm{A}_{i}$ and let $\omega^{2}=\omega_{\pi_{n+2}} \omega_{\pi_{n+1}}$. Since $\omega_{\pi_{i}}$ is unramified, the square root exists, although not uniquely. However, there exists a unique unramified character $\chi$ such that $\chi_{i}^{2}=\omega_{\pi_{i}}$, which is the $\omega_{\pi_{i}}$ we choose. Denote $\nu$ as the similitude character discussed in Section 2.1. Recall, $\nu: \mathrm{G}_{i} \rightarrow \mathrm{GL}_{1}$. Put

$$
\mathcal{P}\left(\phi_{n+2}, \phi_{n+1}\right):=\int_{\mathrm{A}_{n+1} \backslash \mathrm{G}_{n+1}} \Phi_{\phi_{n+2}}(g) \Phi_{\phi_{n+1}}(g) \omega^{-1}(\nu(g)) d g .
$$

Next we establish the convergence of the integral assuming each $\pi_{i}$ is tempered.

\subsection{Convergence of the Integral}

In this section, we first introduce some definitions and prove a couple of technical lemmas before proceeding to the proof of Proposition 3.3. 
Let $\mathrm{G}$ be a reductive group and fix a special maximal compact subgroup $K$ of $\mathrm{G}$. Then we have a Cartan decomposition $\mathrm{G}=K M_{\min }^{+} K$ where

$$
M_{\min }^{+}:=\left\{m \in M_{\min }:\left|\alpha_{i}(m)\right| \leq 1 \text { for } i=1, \ldots, r\right\}
$$

Let $\operatorname{Hom}\left(\mathrm{G}, \mathbb{C}^{\times}\right)$be the group of continuous homomorphisms from $\mathrm{G}$ into $\mathbb{C}^{\times}$, and let $\chi \in \operatorname{Rat}(G)$ be such that $|\chi|_{F} \in \operatorname{Hom}\left(\mathrm{G}, \mathbb{C}^{\times}\right)$is the character defined by

$$
|\chi|_{F}(g)=|\chi(g)|_{F}
$$

Define

$$
G^{1}:=\bigcap_{\chi \in \operatorname{Rat}(G)} \operatorname{Ker}|\chi|_{F}
$$

Set $M_{\min }^{1}:=M_{\min } \cap G^{1}$. From equation (4) of [Wal03],

$$
\mathrm{G}=\coprod_{m \in M_{\min }^{+} / M_{\min }^{1}} K m K
$$

This leads to the following lemma:

Lemma 3.1. For $f \in L^{1}(\mathrm{G})$,

$$
\int_{\mathrm{G}} f(g) d g=\int_{M_{\min }^{+}} \mu(m) \int_{K \times K} f\left(k_{1} m k_{2}\right) d k_{1} d k_{2} d m
$$

where $\mu(m)=A \cdot \operatorname{Vol}(K m K)$ for some constant $A$.

Proof. Let $f \in C_{c}(\mathrm{G})$, we have ([Sil79, pg. 149])

$$
\begin{aligned}
\int_{\mathrm{G}} f(x) d x & =\sum_{m \in M_{\min }^{+} / M_{\min }^{1}} \int_{K m K} f(x) d x \\
& =\sum_{m \in M_{\min }^{+} / M_{\min }^{1}} \int_{K \times K} \operatorname{Vol}(K m K) f\left(k_{1} m k_{2}\right) d k_{1} d k_{2} \\
& =\sum_{m \in M_{\min }^{+} / M_{\min }^{1}} \operatorname{Vol}(K m K) \int_{K \times K} f\left(k_{1} m k_{2}\right) d k_{1} d k_{2}
\end{aligned}
$$




$$
=\int_{M_{\min }^{+} / M_{\min }^{1}} \operatorname{Vol}(K m K) \int_{K \times K} f\left(k_{1} m k_{2}\right) d k_{1} d k_{2} .
$$

Let $\widetilde{F}: M_{\min }^{+} \rightarrow \mathbb{C}$ be such that

$$
\int_{M_{\min }^{+}} \widetilde{F}(m) d m=\int_{M_{\min }^{+} / M_{\min }^{1}} \operatorname{Vol}(K m K) \int_{K \times K} f\left(k_{1} m k_{2}\right) d k_{1} d k_{2} .
$$

We also have that

$$
\int_{M_{\min }^{+}} \widetilde{F}(m) d m=\int_{M_{\min }^{+} / M_{\min }^{1}} \int_{M_{\min }^{1}} \widetilde{F}\left(m m_{1}\right) d m d m_{1}
$$

Then $\widetilde{F}$ is such that

$$
\int_{M_{\min }^{1}} \widetilde{F}\left(m m_{1}\right) d m_{1}=\operatorname{Vol}(K m K) \int_{K \times K} f\left(k_{1} m k_{2}\right) d k_{1} d k_{2} .
$$

Next, we verify

$$
\tilde{F}(m)=\frac{\operatorname{Vol}(K m K)}{\operatorname{Vol}\left(M_{\min }^{1}\right)} \int_{K \times K} f\left(k_{1} m k_{2}\right) d k_{1} d k_{2}
$$

by noting that $M_{\min }^{1}$ is a compact subset of $K$ and considering

$$
\begin{aligned}
\int_{M_{\min }^{1}} \tilde{F}\left(m m_{1}\right) d m_{1} & =\int_{M_{\min }^{1}} \frac{\operatorname{Vol}\left(K m m_{1} K\right)}{\operatorname{Vol}\left(M_{\min }^{1}\right)} \int_{K \times K} f\left(k_{1} m m_{1} k_{2}\right) d k_{1} d k_{2} \\
& =\int_{M_{\min }^{1}} \frac{\operatorname{Vol}(K m K)}{\operatorname{Vol}\left(M_{\min }^{1}\right)} \int_{K \times K} f\left(k_{1} m k_{2}\right) d k_{1} d k_{2} \\
& =\int_{M_{\min }^{1}} d m_{1} \cdot\left(\frac{\operatorname{Vol}(K m K)}{\operatorname{Vol}\left(M_{\min }^{1}\right)} \int_{K \times K} f\left(k_{1} m k_{2}\right) d k_{1} d k_{2}\right) \\
& =\operatorname{Vol}(K m K) \int_{K \times K} f\left(k_{1} m k_{2}\right) d k_{1} d k_{2} .
\end{aligned}
$$

which agrees with (3.2) when $F$ is non-archimedean. Since $M_{\min }^{1}$ is compact, $\operatorname{Vol}\left(M_{\min }^{1}\right)$ is finite. Letting $\mu(m)=A \cdot \operatorname{Vol}(K m K)$, when $F$ is non-archimedean the proof is complete.

In the archimedean case, the same integral formula holds for a non-negative function $\mu(m)$ on $M_{\min }^{+}([$Hel00, Th. 5.8]). 
We introduce the final pieces of general theory needed for the proof of Proposition 3.8. Fix a suitable embedding $\eta: \mathrm{G} \rightarrow \mathrm{GL}_{m}$. Then the height function $\sigma(g)$ with respect to $\eta$ is given by

$$
\sigma(g)=\max _{1 \leq i, j \leq m}\left(\log \left|\eta(g)_{i j}\right|, \log \left|\eta\left(g^{-1}\right)_{i j}\right|\right)
$$

Let $\Xi(g)$ be Harish-Chandra's spherical function on G given by

$$
\Xi(g):=\int_{K} h_{0}(k g) d k
$$

where $h_{0} \in \operatorname{Ind}_{P_{\min }^{\mathrm{G}}}^{G} \mathbb{1}$ is the function that is identically 1 on $K$.

Now we focus our attention on GSpin . Recall that

$$
G^{1}:=\bigcap_{\chi \in \operatorname{Rat}(G)} \operatorname{Ker}|\chi|_{F}
$$

In our case,

$$
G^{1}=\{g \in \text { GSpin }:|\nu(g)|=1\}
$$

Lemma 3.2. Let $\mathrm{G}=\mathrm{GSpin}(V)$ and let $\mathrm{A}$ be the identity component of $\mathrm{G}$. Let $f: \mathrm{G} \rightarrow \mathbb{C}$ be such that $f(z g)=f(g)$. Then $\int_{\mathrm{A} \backslash \mathrm{G}} f(g) d g$ converges if and only if $\int_{G^{1}} f(g) d g$ converges.

Proof. Let A be the identity component of the center of G. Let $p r$ be the natural projection homomorphism from $\mathrm{A} \backslash \mathrm{G}$ to $G^{1} \mathrm{~A} \backslash \mathrm{G}$ sending $g \mapsto g^{\prime} g$. Note that

$$
\begin{aligned}
\text { Ker } p r & =\left\{g \in \mathrm{A} \backslash \mathrm{G}: \operatorname{pr}(g)=1 G^{1} \mathrm{~A}\right\} \\
& =\left\{g \in \mathrm{A} \backslash \mathrm{G}: g^{\prime} g G^{1} \mathrm{~A}=1 G^{1} \mathrm{~A}\right\} \\
& =\left\{g \in \mathrm{A} \backslash \mathrm{G}: g^{\prime} g \in G^{1} \mathrm{~A}\right\} \\
& =\left(G^{1} \cap \mathrm{A}\right) \backslash G^{1} .
\end{aligned}
$$


Hence,

$$
G^{1} \mathrm{~A} \backslash \mathrm{G} \cong\left(\left(G^{1} \cap \mathrm{A}\right) \backslash G^{1}\right) \backslash(\mathrm{A} \backslash \mathrm{G})
$$

Since $\mathrm{A} \cong F^{\times}$, we see that $\mathrm{A} \cap G^{1} \cong \mathcal{O}^{\times}$, which is compact. Moreover, since $G^{1} \mathrm{~A} \backslash \mathrm{G}$ is finite, $\int_{G^{1}} f(g) d g$ converges if and only if $\int_{\mathrm{A} \backslash \mathrm{G}} f(g) d g$ converges.

We utilize the notation from Section 2.1. Denote by $r$ the the Witt rank. Let $P_{\min }=M_{\min } N_{\min }$ be the minimum parabolic subgroup. The Levi factor $M_{\min }$ is isomorphic to the direct product of $\left(F^{\times}\right)^{r} \times \operatorname{GSpin}_{V^{a n}}$. The split component $A_{\min }$ of $M_{\text {min }}$ is isomorphic to $\left(F^{\times}\right)^{r}$ and the Weyl group $W\left(\operatorname{GSpin}(V), A_{\min }\right)$ is of type $B$ if $d$ is odd and is of type $D$ if $d$ is even. Denote an element $A_{\min }$ by $x=\left(x_{1}, x_{2}, x_{3}, \ldots, x_{r}\right)$. The simple roots of $\left(P_{\min }, A_{\min }\right)$ are given by

$$
\alpha_{1}(x)=x_{1} x_{2}^{-1}, \ldots, \alpha_{r-1}(x)=x_{r-1} x_{r}^{-1}
$$

and

$$
\alpha_{r}= \begin{cases}x_{r} & \text { if } d \text { is odd } \\ x_{r-1} x_{r} & \text { if } d \text { is even. }\end{cases}
$$

Let $\delta_{P_{\min }}(x)$ be the modulus character of $P_{\min }$. Then

$$
\delta_{P_{\min }}(x)=\prod_{i=1}^{r}\left|x_{i}\right|^{d+2 r-2 i} .
$$

We define,

$$
A_{\min }^{+, G^{1}}:=\left\{a \in A_{\min }:\left|\alpha_{i}(a)\right| \leq 1 \text { for } i=1, \ldots, r \text { and } a \in G^{1}\right\}
$$

We are now ready to prove the following:

Proposition 3.3. If $\pi_{n+1}$ and $\pi_{n+2}$ are tempered, then the local integral

$$
\mathcal{P}\left(\phi_{n+2}, \phi_{n+1}\right)=\int_{\overline{G_{n+1}}} \Phi_{\phi_{n+2}}(g) \Phi_{\phi_{n+1}}(g) \omega^{-1}(\nu(g)) d g
$$


is absolutely convergent and $\mathcal{P}\left(\phi_{n+2}, \phi_{n+1}\right) \geq 0$ for any $K_{i}$-finite vector $\phi_{n+1} \in \pi_{n+1}$ and $\phi_{n+2} \in \pi_{n+2}$.

Proof. Let $\pi_{n+1}$ and $\pi_{n+2}$ be irreducible tempered representations of $\mathrm{G}_{n+1}$ and $\mathrm{G}_{n+2}$, respectively. Let $\Phi_{\phi_{i}}$ be a matrix coefficient of a tempered representation on $\mathrm{G}_{i}$ defined above. By Lemma 3.2,

$$
\mathcal{P}\left(\phi_{n+2}, \phi_{n+1}\right)=\int_{\overline{G_{n+1}}}\left|\Phi_{\phi_{n+2}}(g)\right|\left|\Phi_{\phi_{n+1}}(g)\right|\left|\omega^{-1}(\nu(g))\right| d g
$$

converges if and only if

$$
\int_{G^{1}}\left|\Phi_{\phi_{n+2}}(g)\right|\left|\Phi_{\phi_{n+1}}(g)\right|\left|\omega^{-1}(\nu(g))\right| d g
$$

converges. Note that $\left|\omega^{-1}(\nu(g))\right|=1$. Using (3.1) and that $G^{1}$ is a subset of $\mathrm{G}_{n+1}$ and $K$ is a compact subset of $G^{1}$ one has the following:

$$
G^{1}=\left(\coprod_{m \in M_{\min }^{+} / M_{\min }^{1}} K m K\right) \bigcap G^{1}=\coprod_{m \in\left(M_{\min }^{+} / M_{\min }^{1}\right) \cap G^{1}} K m K .
$$

We define

$$
M_{\min }^{+, G^{1}}:=\left\{m \in M_{\min }:\left|\alpha_{i}(m)\right| \leq 1 \text { for } i=1, \ldots, r \text { and } m \in G^{1}\right\}
$$

By Lemma 3.1, the convergence of the integral is reduced to the convergence of

$$
\int_{M_{\min }^{+, G^{1}}} \mu(m) \int_{K \times K}\left|\Phi_{\phi_{n+2}}\left(k_{1} m k_{2}\right)\right|\left|\Phi_{\phi_{n+1}}\left(k_{1} m k_{2}\right)\right| d k_{1} d k_{2} d m
$$

where $\mu(m)=A \cdot \operatorname{Vol}(K m K)$. Since $\Phi_{\phi_{n+1}}$ and $\Phi_{\phi_{n+2}}$ are matrix coefficients of tempered representations, they satisfy for any $g \in G$

$$
\left|\Phi_{\phi_{i}}(g)\right| \leq A \Xi(g)(1+\sigma(g))^{B}
$$


for some positive constants $A$ and $B$ [Wal03, pg. 274]. Hence, the convergence of the integral reduces to the convergence of

$$
\int_{M_{\min }^{+, G^{1}}} \mu(m) \int_{K \times K} \Xi_{n+2}(m) \Xi_{n+1}(m)(1+\sigma(m))^{2 B} d k_{1} d k_{2} d m
$$

for $m \in M_{\min }^{+, G^{1}}$.

Per Theorem 4.2.1 in [Sil79, p.154] and [Wal03, Lemma II.1.1], there exists positive constants $A$ and $B$ such that

$$
A^{-1} \delta_{P \min }^{1 / 2}(m) \leq \Xi(m) \leq A \delta_{P \min }^{1 / 2}(m)(1+\sigma(m))^{B}
$$

for any $m$ in $M_{\min }^{+, G^{1}}$. So the convergence of the integral is reduced to the convergence of

$$
\int_{M_{\min }^{+, G^{1}}} \mu(m) \int_{K \times K}\left(\delta_{n+2}\right)^{1 / 2}(m)\left(\delta_{n+1}\right)^{1 / 2}(m)(1+\sigma(m))^{2 B} d k_{1} d k_{2} d m .
$$

But $K \times K$ is compact. Moreover, there exists a positive constant $A$ such that $\mu(m) \leq A \delta_{n}^{-1}$ for any $m \in M_{\text {min }}^{+, 1}$ [Wal03, page 241]. So it is enough to show that

$$
\int_{M_{\min }^{+, G^{1}}}\left(\delta_{n+1}\right)^{-1 / 2}(m)\left(\delta_{n+2}\right)^{1 / 2}(m)(1+\sigma(m))^{2 B} d m
$$

converges.

When $W\left(\mathrm{G}_{n+1}, A_{n+1, \min }\right)$ is of type $B$, then $A_{n+1, \min }^{+} \cap G^{1}$ sits inside of $A_{n+2, \text { min }}^{+} \cap G^{1}$. Thus the integral is reduced to the convergence of

$$
\int_{A_{n+1, \min }^{+, G^{1}}}\left(\delta_{n+1}\right)^{-1 / 2}(m)\left(\delta_{n+2}\right)^{1 / 2}(m)(1+\sigma(m))^{2 B} d m .
$$

GSpin has an extra root dealing with the similitude factor. But $|\nu(g)|=1$, for all $g \in G^{1}$, so it has little effect in the following calculation. Hence, the convergence of the integral is reduced to

$$
\int_{\left|x_{1}\right| \leq \cdots \leq\left|x_{r_{n+1}}\right| \leq\left|x_{0}\right| \leq 1}\left|x_{1} \cdots x_{r_{n+1}}\right|^{1 / 2}\left(1-\sum_{j=1}^{r_{n+1}} \log \left|x_{j}\right|\right)^{2 B} d^{\times} x_{1} \cdots d^{\times} x_{r_{n+1}} d^{\times} x_{0} .
$$


One can show that this integral converges following a result from calculus.

For the second case, when we are in type $D, A_{n+1, \min }^{+} \cap G^{1}$ is not a subset of $A_{n+2, \min }^{+} \cap G^{1}$. Hence, the convergence of the integral in this case is reduced to the convergence of

$$
\begin{aligned}
\int_{\left|x_{1}\right| \leq \cdots \leq\left|x_{r_{n+1}}\right| \leq\left|x_{0}\right| \leq 1} \mid & \left.x_{1} \cdots x_{r_{n+1}}\right|^{1 / 2}\left(1-\sum_{j=1}^{r_{n+1}} \log \left|x_{j}\right|\right)^{2 B} d^{\times} x_{1} \cdots d^{\times} x_{r_{n+1}} d^{\times} x_{0} \\
& +\int_{\left|x_{1}\right| \leq \cdots \leq\left|x_{r_{n+1}-1}\right| \leq\left|x_{r_{n+1}}\right|^{-1} \leq\left|x_{0}\right| \leq 1}\left|x_{1} \cdots x_{r_{n+1}-1} x_{r_{n+1}}^{-1}\right|^{1 / 2} \\
& \left(1-\sum_{j=1}^{r_{n+1}-1} \log \left|x_{j}\right|+\log \left|x_{r_{n+1}}\right|\right)^{2 B} d^{\times} x_{1} \cdots d^{\times} x_{r_{n+1}} d^{\times} x_{0} .
\end{aligned}
$$

This integral can be shown to be convergent similarly to the one above.

Just as in [II10], the second part of the proposition, that $\mathcal{P}\left(\phi_{n+2}, \phi_{n+1}\right) \geq 0$ for any $K_{i}$-finite vector $\phi_{i} \in \pi_{i}$, follows from a general theorem from He [He03, Theorem 2.1]. Recall that we have the isomorphism $\mathrm{A}_{n+1} \backslash \mathrm{G}_{n+1} \cong \mathrm{SO}_{n+1}$. Let $\Xi_{i}$ be HarishChandra spherical functions for $\mathrm{G}_{i}$. By the first part of the proposition,

$$
g_{n+1} \rightarrow \Xi_{n+2}\left(g_{n}\right) \Xi_{n+1}\left(g_{n+1}\right) \omega^{-1}\left(\nu\left(g_{n+1}\right)\right)
$$

belongs to $L^{1}\left(\mathrm{~A}_{n+1} \backslash \mathrm{G}_{n+1}\right)$. Note that Harish-Chandra's spherical function is a matrix coefficient of a tempered representation. Then using He's theorem $\mathcal{P}\left(\phi_{n+2}, \phi_{n+1}\right) \geq$ 0 for any $K_{i}$-finite vector $\phi_{i} \in \pi_{i}$.

\subsection{Calculation of integrals in the unramified sec- tion}

For the remainder of this chapter, we assume $v \notin S$ and we explicitly calculate two integrals, $\zeta(\Xi, \xi)$ and $S_{\Xi^{-1}, \xi^{-1}}(1)$, for such $v$. The product of $\zeta(\Xi, \xi)$ and $S_{\Xi^{-1}, \xi^{-1}}(1)$ is 
the integral that we desire. Away from places in $S$, we are in either the split case, or the quasi-split but non-split case. We compute $\zeta(\Xi, \xi)$ and $S_{\Xi^{-1}, \xi^{-1}}(1)$ in each case.

Let $E$ be the discriminant field. By class field theory, $E$ is either equal to $F$ or the unramified quadratic extension of $F$. Let $q$ be the number of elements of the residue field of $F$. The local zeta function is

$$
\zeta(s):=\frac{1}{1-q^{-s}}
$$

Set $l_{i}:=\lfloor i / 2\rfloor$. Let $\xi_{1}, \ldots \xi_{l_{n+1}}$ and $\Xi_{1}, \ldots \Xi_{l_{n+2}}$ be unramified characters of $F^{\times}$.

Let $B_{i}=T_{i} N_{i}$ be the Borel subgroups where the $N_{i}$ are unipotent radicals and the $T_{i}$ is a maximal torus of $\mathrm{G}_{i}$. Then we can view $\xi$ and $\Xi$ as characters of $B_{n+1}$ and $B_{n+2}$ by extending them by 1 to $N_{i}$. Let $\mathcal{A}_{i} \subset T_{i}$ be the maximal split torus. Without loss of generality, assume $N_{i} \subset N_{i+1}$ and $\mathcal{A}_{i} \subset \mathcal{A}_{i+1}$. Away from $S$, let

$$
\begin{aligned}
& \pi_{n+1}=\operatorname{Ind}_{B_{n+1}}^{\mathrm{G}_{n+1}}(\xi), \\
& \pi_{n+2}=\operatorname{Ind}_{B_{n+2}}^{\mathrm{G}_{n+2}}(\Xi)
\end{aligned}
$$

be unramified principal series of $\mathrm{G}_{n+1}$ and $\mathrm{G}_{n+2}$ respectively.

Choose the $\mathcal{B}_{\pi_{i}}$ so that $\Phi_{f_{\pi_{i}}}$ are the spherical matrix coefficients normalized so that $\Phi_{f_{\pi_{i}}}\left(k_{i}\right)=1$ for all $k_{i} \in K_{i}$. Then we have

$$
\Phi_{\pi_{\pi_{i}}}\left(g_{i}\right)=\int_{K_{i}} f_{\pi_{i}}\left(k_{i} g_{i}\right) d k_{i}
$$

for all $g_{i} \in \mathrm{G}_{i}$. Denote $\overline{\mathrm{G}}_{i}=\mathrm{A}_{i} \backslash \mathrm{G}_{i}$. Consider the local integral

$$
F\left(g_{n+2}\right)=\int_{\overline{\mathrm{G}_{n+1}}} \Phi_{f_{\pi_{n+2}}}\left(g_{n+2}^{-1} g_{n+1}\right) \Phi_{f_{\pi_{n+1}}}\left(g_{n+1}\right) \omega^{-1}\left(\nu\left(g_{n+2}^{-1} g_{n+1}\right)\right) d g_{n+1} \text {. }
$$

Next, we review the theory of Shintani functions. Kato-Murase-Sugano [KMS03] showed that if both $\mathrm{G}_{n+1}$ and $\mathrm{G}_{n+2}$ are split, then there exists a Shintani function. 
We will show in a moment that $F\left(g_{n+2}\right)$ is indeed a Shintani function. Although the result is for the orthogonal groups, the argument follows for $A_{i} \backslash G_{i}$. Let $\mathcal{H}_{i}$ denote the Hecke algebra $\mathcal{H}\left(K_{i} \backslash \mathrm{G}_{i} / K_{i}\right)$. Denote $\omega_{i}$ the algebra homomorphism given by the Satake isomorphism from $\mathcal{H}_{i}$ to the complexes.

A Shintani function $S$ is a function that satisfies

1. $\mathcal{L}\left(k_{n+1}\right) \mathcal{R}\left(k_{n+2}\right) S=S$ for any $k_{n+2} \in K_{n+2}$ and $k_{n+1} \in K_{n+1}$,

2. $\mathcal{L}\left(\phi_{n+1}\right) \mathcal{R}\left(\phi_{n+2}\right) S=\omega_{\pi_{n+1}}\left(\phi_{n+1}\right) \omega_{\pi_{n+2}}\left(\phi_{n+2}\right) S$ for any $\phi_{n+1} \in \mathcal{H}_{n+1}$ and $\phi_{n+2} \in$ $\mathcal{H}_{n+2}$.

Lemma 3.4. Let $F$ be as (3.3). Then $F$ is a Shintani function for $\tilde{\pi}_{n+2}$ and $\tilde{\pi}_{n+1}$.

Proof. We begin by verifying the first condition.

$$
\begin{aligned}
& \mathcal{L}\left(k_{n+1}\right) \mathcal{R}\left(k_{n+2}\right) F\left(g_{n+2}\right)=\int_{\bar{G}_{n+1}} \Phi_{f_{\pi_{n+2}}}\left(k_{n+2}^{-1} g_{n+2}^{-1} k_{n+1} g_{n+1}\right) \Phi_{f_{\pi_{n+1}}}\left(g_{n+1}\right) \\
& \cdot \omega^{-1}\left(\nu\left(k_{n+2}^{-1} g_{n+2}^{-1} k_{n+1} g_{n+1}\right)\right) d g_{n+1} \\
&=\int_{\bar{G}_{n+1}} \Phi_{f_{\pi_{n+2}}}\left(k_{n+2}^{-1} g_{n+2}^{-1} g_{n+1}\right) \Phi_{f_{\pi_{n+1}}}\left(k_{n+1}^{-1} g_{n+1}\right) \\
&=\int_{\bar{G}_{n+1}} \int_{K_{n+2}} f_{\pi_{n+2}}\left(k _ { n + 2 } ^ { \prime } k _ { n + 2 } ^ { - 1 } \left(\nu\left(k_{n+2}^{-1} g_{n+2}^{-1} g_{n+1}\right)\right.\right. \\
&\left.=\int_{\bar{G}_{n+1}} \Phi_{f_{\pi_{n+2}}}\left(g_{n+2}^{-1} g_{n+1} g_{n+1}\right)\right) d g_{n+1} \Phi_{\pi_{\pi_{n+1}}}\left(g_{n+2}\right) \omega^{-1}\left(\nu\left(g_{n+2}^{-1} g_{n+1}\right) d g_{n+1}\right. \\
&=F\left(g_{n+2}\right) .
\end{aligned}
$$

Next, we verify the second condition.

$$
\mathcal{L}\left(\phi_{n+1}\right) \mathcal{R}\left(\phi_{n+2}\right) F\left(g_{n+2}^{\prime}\right)=\int_{G_{n+1}} \mathcal{R}\left(\phi_{n+1}\right) F\left(g_{n+1}^{-1} g_{n+2}^{\prime}\right) \phi_{n+1}\left(g_{n+1}\right) d g_{n+1}
$$




$$
\begin{aligned}
& =\int_{\bar{G}_{n+2}} \int_{\bar{G}_{n+1}} F\left(g_{n+1}^{-1} g_{n+2}^{\prime} g_{n+2}\right) \phi_{n+1}\left(g_{n+1}\right) \phi_{n+2}\left(g_{n+2}\right) d g_{n+1} d g_{n+2} \\
& =\int_{\bar{G}_{n+2}} \int_{\bar{G}_{n+1}} \int_{\bar{G}_{n+1}} \Phi_{f_{\pi_{n+2}}}\left(g_{n+2}^{-1} g_{n+2}^{\prime-1} g_{n+1} g_{n+1}^{\prime}\right) \Phi_{f_{\pi_{n+1}}}\left(g_{n+1}^{\prime}\right) \\
& \text { - } \phi_{n+1}\left(g_{n+1}\right) \phi_{n+2}\left(g_{n+2}\right) \omega^{-1}\left(\nu\left(g_{n+2}^{-1} g_{n+2}^{-1} g_{n+1} g_{n+1}^{\prime}\right) d g_{n+1} d g_{n+2}\right. \\
& =\int_{\bar{G}_{n+2}} \int_{\bar{G}_{n+1}} \int_{\bar{G}_{n+1}}\left(\int_{K_{n+2}} f_{\pi_{n+2}}\left(k_{n+2} g_{n+2}^{-1} g_{n+2}^{\prime-1} g_{n+1} g_{n+1}^{\prime}\right) d k_{n+2}\right. \\
& \left.\cdot \int_{K_{n+1}} f_{\pi_{n+1}}\left(k_{n+1} g_{n+1}^{\prime}\right) d k_{n+1}\right) \phi_{n+1}\left(g_{n+1}\right) \phi_{n+2}\left(g_{n+2}\right) \\
& \cdot \omega^{-1}\left(\nu\left(g_{n+2}^{-1} g_{n+2}^{\prime-1} g_{n+1} g_{n+1}^{\prime}\right) d k_{n+1} d g_{n+1} d g_{n+2}\right. \\
& =\int_{\bar{G}_{n+1}} \int_{K_{n+2}} \int_{\bar{G}_{n+2}} f_{\pi_{n+2}}\left(k_{n+2} g_{n+2}^{-1} g_{n+2}^{\prime} g_{n+1} g_{n+1}^{\prime}\right) \phi_{n+2}\left(g_{n+2}\right) d g_{n+2} d k_{n+2} \\
& \cdot \int_{K_{n+1}} \int_{\bar{G}_{n+1}} f_{\pi_{n+1}}\left(k_{n+1} g_{n+1}^{\prime}\right) \phi_{n+1}\left(g_{n+1}\right) d g_{n+1} d k_{n+1} d g_{n+1} \\
& \cdot \omega^{-1}\left(\nu\left(g_{n+2}^{-1} g_{n+2}^{\prime-1} g_{n+1} g_{n+1}^{\prime}\right) d g_{n+1} d k_{n+1}\right. \\
& =\int_{\bar{G}_{n+1}} \int_{K_{n+2}} \int_{\bar{G}_{n+2}} f_{\pi_{n+2}}\left(k_{n+2} g_{n+2}^{\prime} g_{n+1}\right) \phi_{n+2}\left(g_{n+2}\right) d g_{n+2} d k_{n+2} \\
& \cdot \int_{K_{n+1}} \int_{\bar{G}_{n+1}} f_{\pi_{n+1}}\left(k_{n+1} g_{n+1}^{\prime}\right) \phi_{n+1}\left(g_{n+1}\right) d g_{n+1} d k_{n+1} d g_{n+1} \\
& \cdot \omega^{-1}\left(\nu\left(g_{n+2}^{-1} g_{n+2}^{\prime-1} g_{n+1} g_{n+1}^{\prime}\right) d g_{n+1} d k_{n+1}\right. \\
& =\int_{\bar{G}_{n+1}} \int_{K_{n+2}} \int_{\bar{G}_{n+2}} f_{\pi_{n+2}}\left(k_{n+2} g_{n+2}^{\prime} g_{n+1}\right) \phi_{n+2}\left(g_{n+2}\right) d g_{n+2} d k_{n+2} \\
& \int_{K_{n+1}} \int_{\bar{G}_{n+1}} f_{\pi_{n+1}}\left(k_{n+1} g_{n+1} g_{n+1}\right) \phi_{n+1}\left(g_{n+1}\right) d g_{n+1} d k_{n+1} d g_{n+1} \\
& \cdot \omega^{-1}\left(\nu\left(g_{n+2}^{\prime-1} g_{n+1}\right) d g_{n+1} d k_{n+1}\right. \\
& =\omega_{\pi_{n+1}}\left(\phi_{n+1}\right) \omega_{\pi_{n+2}}\left(\phi_{n+2}\right) \int_{\bar{G}_{n+2}} \int_{\bar{G}_{n+1}} \int_{K_{n+2}} f_{\pi_{n+2}}\left(k_{n+2}^{\prime} g_{n+2}\right) \\
& \cdot \int_{K_{n+1}} \int_{\bar{G}_{n+1}} f_{\pi_{n+1}}\left(k_{n+1} g_{n+1}\right) \omega^{-1}\left(\nu\left(g_{n+2}^{-1} g_{n+1}\right) d g_{n+1} d k_{n+1}\right. \\
& =\omega_{\pi_{n+1}}\left(\phi_{n+1}\right) \omega_{\pi_{n+2}}\left(\phi_{n+2}\right) F\left(g_{n+2}\right) \text {. }
\end{aligned}
$$

Hence, $F$ is a Shintani function.

We need the following two lemmas before proceeding. 
Lemma 3.5. There exists a unique open orbit $B_{n+2} \backslash \mathrm{G}_{n+2} / B_{n+2}$ which has a representative $\eta_{n+2} \in K_{n+2}$.

Proof. Note that $B_{n+2} \backslash \mathrm{G}_{n+2} / B_{n+1}=\left\{B_{n+2} g B_{n+1}: g \in \mathrm{G}_{n+2}\right\}$. Clearly, $B_{n+2} g B_{n+1}$ is a subset of $\mathrm{G}_{n+2}$. Let $W$ denote the Weyl group of $\mathrm{G}_{n+2}$ and let $N$ be the unipotent radical of $G$. Then we have the Bruhat decomposition as follows:

$$
\begin{aligned}
\mathrm{G}_{n+2}= & \bigsqcup_{w \in W} B_{n+2} w B_{n+2} \\
& =\bigcup_{w \in W} B_{n+2} w T_{n+2} N_{n+2} \quad\left(B_{n+2}=T_{n+2} N_{n+2}\right) \\
& =\bigcup_{w \in W} B_{n+2} w T_{n+2} w^{-1} w N_{n+2} \quad\left(w T_{n+2} w^{-1}=T_{n+2}\right) \\
& =\bigcup_{w \in W} B_{n+2} w N_{n+2} .
\end{aligned}
$$

If $w$ is the longest Weyl element, then $B_{n+2} w N_{n+2}$ is open and dense. Note that $B_{n+2} w N_{n+2}=B_{n+2} w B_{n+2} \supsetneq B_{n+2} w B_{n+1}$ The unipotent radical for GSpin is the same as for SO so the argument in Cor 7.1 in [GPSR97] holds for GSpin which concludes the proof.

Lemma 3.6. Let $\mathrm{A}_{n+1}$ be the identity component of $\mathrm{G}_{n+1}$ and let $\eta_{n+2} \in K_{n+2}$. Then

$$
\eta_{n+2}^{-1} B_{n+2} \eta_{n+2} \cap B_{n+1}=\mathrm{A}_{n+1}
$$

Proof. First, to see that

$$
\mathrm{A}_{n+1} \subseteq \eta_{n+2}^{-1} B_{n+2} \eta \cap B_{n+1}
$$

let $Z\left(\mathrm{G}_{i}\right)$ denote the center of $\mathrm{G}_{i}$. Using Proposition 2.3 in [AS06], $\mathrm{A}_{n+2}=\mathrm{A}_{n+1} \subseteq$ $Z\left(\mathrm{G}_{n+1}\right)=Z\left(\mathrm{G}_{n+1}\right) \cap Z\left(\mathrm{G}_{n+2}\right)=Z\left(B_{n+1}\right) \cap Z\left(B_{n+2}\right) \subseteq B_{n+1} \cap \eta_{n+2}^{-1} B_{n+2} \eta_{n+2}$. Here we also use Corollary 6.4.6 in [Spr98] which shows the center of a group equals the center of the Borel subgroup. The last inclusion comes from $Z\left(B_{n+1}\right) \subseteq B_{n+1}$ and $Z\left(B_{n+2}\right) \subseteq \eta^{-1} B_{n+2} \eta_{n+2}$. Hence, $\mathrm{A}_{n+1} \subseteq \eta^{-1} B_{n+2} \eta_{n+2} \cap B_{n+1}$. 
It remains to show that $\eta_{n+2}^{-1} B_{n+2} \eta \cap B_{n+1} \subseteq A_{n+1}$. Since there exists the surjective map pr taking Borel subgroups in GSpin to Borel subgroups in SO, if $\eta^{-1} B_{n+2} \eta_{n+2} \cap$ $B_{n+1}$ was not the identity component of the center, then this would contradict the claim of Ichino-Ikeda that $\eta^{-1} B_{n+2} \eta_{n+2} \cap B_{n+1}=\{1\}[$ II10, pg. 1389]. Hence,

$$
\mathrm{A}_{n+1}=\eta_{n+2}^{-1} B_{n+2} \eta \cap B_{n+1}
$$

Let $Y_{\Xi, \xi}$ be the function on $\mathrm{G}_{n+2}$ determined by the following conditions:

1. $Y_{\Xi, \xi}\left(b_{n+2} g_{n+2} b_{n+1}\right)=\left(\Xi^{-1} \delta_{n+2}^{1 / 2}\right)\left(b_{n+2}\right)\left(\xi \delta_{n+1}^{-1 / 2}\right)\left(b_{n+1}\right) Y_{\Xi, \xi}\left(g_{n+2}\right) \omega\left(\nu\left(b_{n+2}\right)\right)$ for any $b_{i} \in B_{i}$,

2. $Y_{\Xi, \xi}\left(\eta_{n+2}\right)=1$,

3. $Y_{\Xi, \xi}\left(g_{n+2}\right)=0$ if $g_{n+2} \notin B_{n+2} \eta_{n+2} B_{n+1}$.

Here $\delta_{i}$ is the modulus character for $B_{i}$. Note that $Y_{\Xi, \xi}$ is well-defined. To see this, let $b_{n+2}, c_{n+2} \in B_{n+2}$, and let $z \in A_{n+1}$. Note that if $b_{n+2} \eta_{n+2} b_{n+1}=c_{n+2} \eta_{n+2} c_{n+1}$, then $\eta_{n+2}^{-1} c_{n+2}^{-1} b_{n+2} \eta_{n+2}=c_{n+1} b_{n+1}^{-1}=z$. So we have,

$$
c_{n+1}=z b_{n+1} \text { and } c_{n+2}=z^{-1} b_{n+2} \text {. }
$$

Thus,

$$
\begin{aligned}
& Y_{\Xi, \xi}\left(c_{n+2} \eta c_{n+1}\right)= \Xi^{-1} \delta_{n+2}^{1 / 2}\left(c_{n+2}\right)\left(\xi \delta_{n+1}^{-1 / 2}\right)\left(c_{n+1}\right) \omega\left(v\left(c_{n+2}\right)\right) Y_{\Xi, \xi}\left(\eta_{n+2}\right) \\
&= \Xi^{-1} \delta_{n+2}^{1 / 2}\left(z^{-1} b_{n+2}\right)\left(\xi \delta_{n+1}^{-1 / 2}\right)\left(z b_{n+1}\right) \omega\left(v\left(z^{-1} b_{n+2}\right)\right) Y_{\Xi, \xi}\left(\eta_{n+2}\right) \\
&= \Xi^{-1} \delta_{n+2}^{1 / 2}\left(z^{-1}\right) \Xi^{-1} \delta_{n+2}^{1 / 2}\left(b_{n+2}\right)\left(\xi \delta_{n+1}^{-1 / 2}\right)(z) \xi \delta_{n+1}^{-1 / 2}\left(b_{n+1}\right) \\
& \omega\left(z^{-2}\right) \omega\left(v\left(b_{n+2}\right)\right) Y_{\Xi, \xi}\left(\eta_{n+2}\right)
\end{aligned}
$$




$$
\begin{aligned}
& =\Xi \delta_{n+2}^{-1 / 2}(z) \Xi^{-1} \delta_{n+2}^{1 / 2}\left(b_{n+2}\right)\left(\xi \delta_{n+1}^{-1 / 2}\right)(z) \xi \delta_{n+1}^{-1 / 2}\left(b_{n+1}\right) \\
& \omega(z)^{-2} \omega\left(v\left(b_{n+2}\right)\right) Y_{\Xi, \xi}\left(\eta_{n+2}\right) \\
& =\omega_{\pi_{n+2}}(z) \Xi^{-1} \delta_{n+2}^{1 / 2}\left(b_{n+2}\right) \omega_{\pi_{n+1}}(z) \xi \delta_{n+1}^{-1 / 2}\left(b_{n+1}\right) \omega(z)^{-2} \\
& \quad \omega\left(v\left(b_{n+2}\right)\right) Y_{\Xi, \xi}\left(\eta_{n+2}\right) \\
& =\omega_{\pi_{n+2}}(z) \Xi^{-1} \delta_{2}^{1 / 2}\left(b_{n+2}\right) \omega_{\pi_{n+1}}(z) \xi \delta_{n+1}^{-1 / 2}\left(b_{n+1}\right) \omega_{\pi_{n+2}}(z)^{-1} \\
& \quad \omega_{\pi_{n+1}}(z)^{-1} \omega\left(v\left(b_{n+2}\right)\right) Y_{\Xi, \xi}\left(\eta_{n+2}\right) \\
& =\Xi^{-1} \delta_{n+2}^{1 / 2}\left(b_{n+2}\right) \xi \delta_{n+1}^{1 / 2}\left(b_{n+1}\right) \omega\left(v\left(b_{n+2}\right)\right) Y_{\Xi, \xi}\left(\eta_{n+2}\right) \\
& =Y_{\Xi, \xi}\left(b_{n+2} \eta_{n+2} b_{n+1}\right) .
\end{aligned}
$$

Hence, $Y_{\Xi, \xi}$ is well defined.

Next, we define $l_{\Xi, \xi} \in \operatorname{Hom}_{\mathrm{G}_{n+1}}\left(\pi_{n+2}, \tilde{\pi}_{n+1}\right)$ by

$$
l_{\Xi, \xi}\left(\operatorname{pr}_{1}(f)\right)\left(g_{n+1}\right)=\int_{\mathrm{G}_{n+2}} f\left(g_{n+2} g_{n+1}\right) Y_{\Xi, \xi}\left(g_{n+2}\right) d g_{n+2}, \quad g_{n+1} \in \mathrm{G}_{n+1} .
$$

Since $f$ is compactly supported, the integral converges.

Here, $\operatorname{pr}_{1}: C_{c}^{\infty}\left(\mathrm{G}_{n+1}\right) \rightarrow \pi_{n+2}$ is given by

$$
\operatorname{pr}_{1}(f)\left(g_{n+2}\right)=\int_{B_{n+1}}\left(\Xi_{n+2}^{-1} \delta_{n+2}^{1 / 2}\right)\left(b_{n+2}\right) f\left(b_{n+2} g_{n+2}\right) d b_{n+2}
$$

Let $\langle$,$\rangle be the natural pairing on \pi_{n+1} \times \tilde{\pi}_{n+1}$ defined by

$$
\left\langle\phi_{n+1}, \phi_{n+1}^{\prime}\right\rangle=\int_{K_{n+1}} \phi_{n+1}\left(k_{n+1}\right) \phi_{n+1}^{\prime}\left(k_{n+1}\right) d k_{n+1}
$$

for $\phi_{n+1} \in \pi_{n+1}$ and $\phi_{n+1}^{\prime} \in \tilde{\pi}_{n+1}$.

Choose $f=\mathbb{1}_{K_{n+2}}$, the characteristic function on $K_{n+2}$. Put

$$
S_{\Xi, \xi}\left(g_{n+2}\right)=\left\langle f_{\pi_{n+1}}, l_{\Xi, \xi}\left(\pi_{n+2}\left(g_{n+2}\right) f_{\pi_{n+2}}\right)\right\rangle .
$$


Then we have,

$$
\begin{aligned}
S_{\Xi, \xi}\left(g_{n+2}\right) & =\int_{K_{n+1}} f_{\pi_{n+1}}\left(k_{n+1}\right) l_{\Xi, \xi}\left(\pi_{n+2}\left(g_{n+2}\right) f_{\pi_{n+2}}\right)\left(k_{n+1}\right) d k_{n+1} \\
& =\int_{K_{n+1}} f_{\pi_{n+1}}\left(k_{n+1}\right) \int_{\mathrm{G}_{n+2}} f\left(g_{n+2}^{\prime} k_{n+1} g_{n+2}\right) Y_{\Xi, \xi}\left(g_{n+2}^{\prime}\right) d g_{n+2}^{\prime} d k_{n+1} \\
& =\int_{K_{n+1}} f_{\pi_{n+1}}\left(k_{n+1}\right) \int_{\mathrm{G}_{n+2}} \mathbb{1}_{K_{n+2}}\left(g_{n+2}^{\prime} k_{n+1} g_{n+2}\right) Y_{\Xi, \xi}\left(g_{n+2}^{\prime}\right) d g_{n+2}^{\prime} d k_{n+1} \\
& =\int_{K_{n+1}} f_{\pi_{n+1}}\left(k_{n+1}\right) \int_{\mathrm{G}_{n+2}} \mathbb{1}_{K_{n+2}}\left(g_{n+2}^{\prime} g_{n+2}^{-1} k_{n+1}^{-1} k_{n+1} g_{n+2}\right) \\
& \left.=\int_{K_{n+1}} f_{\pi_{n+1}}\left(k_{n+1}\right) \int_{\mathrm{G}_{n+2}} \mathbb{1}_{K_{n+2}}\left(g_{n+2}^{\prime}\right) Y_{\Xi, \xi}^{\prime}\left(g_{n+2}^{\prime} g_{n+2}^{-1} k_{n+1}^{-1}\right) d g_{n+2}^{\prime} d k_{n+1}^{-1}\right) d g_{n+2}^{\prime} d k_{n+1} \\
& =\int_{K_{n+1}} f_{\pi_{n+1}}\left(k_{n+1}\right) \int_{K_{n+2}} Y_{\Xi, \xi}\left(k_{n+2} g_{n+2}^{-1} k_{n+1}\right) d k_{n+2} d k_{n+1} \\
& =\int_{K_{n+2} \times K_{n+1}} Y_{\Xi, \xi}\left(k_{n+2} g_{n+2}^{-1} k_{n+1}\right) d k_{n+2} d k_{n+1} .
\end{aligned}
$$

Note that $S_{\Xi, \xi}$ is also a Shintani Function. Define

$$
T_{\Xi, \xi}\left(g_{n+2}\right)=\int_{\overline{\mathrm{G}_{n+1}}} f_{\pi_{n+2}}\left(g_{n+2} g_{n+1}\right) f_{\pi_{n+1}}\left(g_{n+1}\right) \omega^{-1}\left(\nu\left(g_{n+2} g_{n+1}\right)\right) d g_{n+1}
$$

if $g_{n+2} \in B_{n+2} \eta_{n+2} B_{n+1}$ and 0 otherwise.

The relationship between $T_{\Xi, \xi}$ and $Y_{\Xi^{-1}, \xi^{-1}}$ is

$$
T_{\Xi, \xi}\left(g_{n+2}\right)=T_{\Xi, \xi}\left(\eta_{n+2}\right) Y_{\Xi^{-1}, \xi^{-1}}\left(g_{n+2}\right) .
$$

To see this, let $g_{n+2} \in B_{n+2} \eta_{n+2} B_{n+1}$ and let $g_{n+2}=b_{n+2} \eta_{n+2} b_{n+1}$. Then we have,

$$
\begin{aligned}
T_{\Xi, \xi}\left(g_{n+2}\right)= & T_{\Xi, \xi}\left(b_{n+2} \eta_{n+2} b_{n+1}\right)=\int_{\overline{\mathrm{G}_{n+1}}} f_{\pi_{n+2}}\left(b_{n+2} \eta_{n+2} b_{n+1} g_{n+1}\right) f_{\pi_{n+1}}\left(g_{n+1}\right) \\
= & \Xi\left(b_{n+2}\right) \delta^{1 / 2}\left(\nu\left(b_{n+2} \eta_{n+2} b_{n+1} g_{n+1}\right)\right) d g_{n+1} \\
& \int_{\overline{\mathrm{G}_{n+1}}} f_{\pi_{n+2}}\left(\eta_{n+2} b_{n+1} g_{n+1}\right) f_{\pi_{n+1}}\left(g_{n+1}\right) \omega^{-1}\left(\nu\left(\eta_{n+2} b_{n+1} g_{n+1}\right)\right) d g_{n+1}
\end{aligned}
$$




$$
\begin{aligned}
= & \Xi\left(b_{n+2}\right) \delta_{n+2}^{1 / 2}\left(b_{n+2}\right) \omega^{-1}\left(\nu\left(b_{n+2}\right)\right) \\
& \int_{\frac{\mathrm{G}_{n+1}}{}} f_{\pi_{n+2}}\left(\eta_{n+2} g_{n+1}\right) f_{\pi_{n+1}}\left(b_{n+1}^{-1} g_{n+1}\right) \omega^{-1}\left(\nu\left(\eta_{n+2} g_{n+1}\right)\right) d g_{n+1} \\
= & \Xi\left(b_{n+2}\right) \delta_{n+2}^{1 / 2}\left(b_{n+2}\right) \omega^{-1}\left(\nu\left(b_{n+2}\right)\right) \xi^{-1}\left(b_{n+1}\right) \delta_{n+1}^{-1 / 2}\left(b_{n+1}\right) \\
& \quad \int_{\overline{\mathrm{G}}_{n+1}} f_{\pi_{n+2}}\left(\eta_{n+2} g_{n+1}\right) f_{\pi_{n+1}}\left(g_{n+1}\right) \omega^{-1}\left(\nu\left(\eta_{n+2} g_{1}\right)\right) d g_{1} \\
= & Y_{\Xi^{-1}, \xi^{-1}}\left(g_{n+2}\right) T_{\Xi, \xi}\left(\eta_{n+2}\right) .
\end{aligned}
$$

Note that $T_{\Xi, \xi}$ does not depend on the choice of representative of $\eta_{n+2}$ so we define $\zeta(\Xi, \xi):=T_{\Xi, \xi}\left(\eta_{n+2}\right)$. Hence, we have

$$
\begin{aligned}
F\left(g_{n+2}\right) & =\int_{\overline{\mathrm{G}_{n+1}}} \int_{K_{n+2}} \int_{K_{n+1}} f_{\pi_{n+2}}\left(k_{n+2} g_{n+2}^{-1} g_{n+1}\right) f_{\pi_{n+1}}\left(k_{n+1} g_{n+1}\right) \\
& \left.=\int_{\overline{\mathrm{G}_{n+1}}} \int_{K_{n+2}} \int_{K_{n+1}} f_{\pi_{n+2}}\left(k_{n+2} g_{n+2}^{-1} k_{n+1}^{-1} g_{n+1}\right)\right) d k_{n+1} d k_{n+2} d g_{n+1} f_{\pi_{n+1}}\left(g_{n+1}\right) \\
& =\int_{\overline{\mathrm{G}_{n+1}}} \int_{K_{n+2}} \int_{K_{n+1}} f_{\pi_{n+2}}\left(k_{n+2} g_{n+2}^{-1} k_{n+1} g_{n+1}\right) f_{\pi_{n+1}}\left(g_{n+1}\right) \\
& =\int_{K_{n+2} \times K_{n+1}} T_{\Xi, \xi}\left(k_{n+2} g_{n+2}^{-1} k_{n+1}\right) d k_{n+2} d k_{n+1} \\
& =T_{\Xi, \xi}\left(\eta_{n+2}\right) \int_{K_{n+2} \times K_{n+1}} Y_{\Xi^{-1}, \xi^{-1}}\left(k_{n+2} g_{n+2}^{-1} k_{n+1}\right) d k_{n+2} d k_{n+1} \\
& \left.\left.=\zeta(\Xi, \xi) S_{\Xi-1} g_{n+2} k_{n+1} g_{n+1}\right)\right) d k_{n+1} d k_{n+2} d g_{n+1} \\
&
\end{aligned}
$$

As in [II10, pg. 1390], $F\left(g_{n+2}\right)$ is convergent for $\Xi, \xi$ sufficiently close to the unitary axis. Indeed, Proposition 3.3 holds for such $\Xi$ and $\xi$. Hence, $T_{\Xi, \xi}\left(k_{n+2} g_{n+2}^{-1} k_{n+1}\right)$ converges for almost all $k_{n+2}, k_{n+1}$ such that $k_{n+2} g_{n+2}^{-1} k_{n+1} \in B_{n+2} \eta_{n+2} B_{n+1}$. Moreover, $T_{\Xi, \xi}$ converges for some $g \in B_{n+2} \eta_{n+2} B_{n+1}$ precisely when it converges for all $g \in B_{n+2} \eta_{n+2} \eta_{n+1}$. Thus, $T_{\Xi, \xi}\left(\eta_{n+2}\right)$ converges. Hence, $S_{\Xi^{-1}, \xi^{-1}}\left(g_{n+2}\right)$ converges as 
well.

\subsubsection{Calculation of $\zeta(\Xi, \xi)$ when $E=F$}

We consider the case when $E$ is equal to $F$, and the residual characteristic of $F$ is not 2. As before, $T_{i}$ is a maximal torus of $G_{i}$. Let $\mathcal{A}_{i}$ be the split component of the Levi factor $M_{i}$. Since we are in the case that each $\mathrm{G}_{i}$ is split, we have that

$$
T_{n+2}=\mathcal{A}_{n+2} \simeq\left(F^{\times}\right)^{\lfloor(n+2) / 2\rfloor} \text { and } T_{n+1}=\mathcal{A}_{n+1} \simeq\left(F^{\times}\right)^{\lfloor(n+1) / 2\rfloor} .
$$

Let $\widetilde{\Xi}, \xi, \Xi$ be characters of $T_{n}, T_{n+1}, T_{n+2}$, respectively, where

$$
\begin{gathered}
\widetilde{\Xi}:=\left(\Xi_{1}, \ldots, \Xi_{\lfloor n / 2\rfloor}, \chi_{0}\right), \\
\xi:=\left(\xi_{1}, \ldots, \xi_{\lfloor(n+1) / 2\rfloor}, \chi_{1}\right), \\
\Xi:=\left(\Xi_{1}, \ldots, \Xi_{\lfloor(n+2) / 2\rfloor}, \chi_{0}\right) .
\end{gathered}
$$

Moreover, denote $l:=\lfloor(n+2) / 2\rfloor$ and let $\chi_{n+1}$ be unramified and such that $\chi_{n+1}^{2}=\omega_{\pi_{n+1}}$. Recall that $\omega^{2}=\omega_{\pi_{n+2}} \omega_{\pi_{n+1}}$. Hence, $\omega^{2}=\left(\chi_{n+1} \chi_{n}\right)^{2}$. Per Asgari [AS06, pg. 178], $\omega^{-1} \chi_{n+1}=\chi_{0}^{-1 / 2}$. We can view $\pi_{n} \otimes \chi_{0}^{-1 / 2}$ as a representation of $\overline{G_{n}} \cong \mathrm{SO}_{n}$. Define $\overline{\pi_{n}}:=\pi_{n} \otimes \chi_{0}^{-1 / 2}$. Take $V_{n}$ to be a $n$-dimensional symmetric space, with associated symmetric form $\langle\cdot, \cdot\rangle_{V_{n}}$.

Let $e, f \in V$ such that $\langle e, e\rangle=0,\langle f, f\rangle=0$, and $\langle e, f\rangle=1$. Then, $V_{n+2}=V_{n} \oplus \operatorname{Span}\{e, f\}$ and $V_{n+1}=V_{n} \oplus \operatorname{Span}\{e+f\}$. Define

$$
W:=V_{n} \oplus V_{n}^{-} \oplus \operatorname{Span}\{e, f\} .
$$

Note that $\operatorname{dim} W=2 n+2$ and so $\mathrm{SO}(W) \cong \mathrm{SO}_{2 n+2}$. Let $Q:=\operatorname{Span}\{e, f\}$. Then,

$$
\overline{\mathrm{G}_{n}}\left(V_{n}\right) \times \overline{\mathrm{G}_{n+1}}\left(V_{n+1}\right) \subseteq \mathrm{SO}(W) .
$$


Choose a basis of $W$ to be

$$
\left\{v_{1} \oplus-v_{1}, v_{2} \oplus-v_{2}, \ldots, v_{n} \oplus-v_{n}, e, v_{1} \oplus v_{1}, v_{2} \oplus v_{2}, \ldots, v_{n} \oplus v_{n}, f\right\}
$$

Hence, $V^{\dagger}=\operatorname{Span}\left\{v_{i} \oplus\left(-v_{i}\right), e\right\}$ where $v_{i} \in V_{n}$ and $-v_{i} \in V_{n}^{-}$. Note that $V^{\dagger}$ is a maximal isotropic subspace.

Denote $P:=\operatorname{Stab}_{\mathrm{SO}(W)} V^{\dagger}$, the corresponding maximal parabolic subgroup. Note that $P$ is the Siegel parabolic in $\mathrm{SO}(W)$. Then $P=M N$, where $M$ is the Levi subgroup and $N$ is the unipotent radical. We have that $M$ is isomorphic to $\operatorname{GL}\left(V^{\dagger}\right)$. Consider the following induced representation of $\mathrm{SO}(W)$ :

$$
I\left(\Xi_{l}\right):=\operatorname{Ind}_{P}^{\mathrm{SO}(W)}\left(\left(\Xi_{l} \otimes \chi_{0}^{-1 / 2}\right) \circ \operatorname{det}_{V^{\dagger}}\right)
$$

where the induction is normalized. Let $f_{0} \in I\left(\Xi_{l}\right)$ be the spherical vector, normalized so that $f_{0}(1)=1$. Define $\tilde{f}_{0}$ to be $f_{0}$ restricted to $\overline{\mathrm{G}_{n}} \times \overline{\mathrm{G}_{n+2}}$. Let $\overline{f_{\pi_{n}}} \in \overline{\pi_{n}}$, and define

$$
\Lambda\left(f_{0}, \overline{f_{\pi_{n}}}\right)\left(g_{n+2}\right):=\int_{\overline{\mathrm{G}_{n}}} \widetilde{f}_{0}\left(g_{n}, g_{n+2}\right) \overline{\pi_{n}}\left(g_{n}\right) \overline{f_{\pi_{n}}} d g_{n}
$$

Hence,

$$
\Lambda\left(f_{0}, \overline{f_{\pi_{n}}}\right)\left(g_{n+2}\right)\left(\overline{g_{n}}\right):=\int_{\overline{\mathrm{G}_{n}}} \widetilde{f}_{0}\left(g_{n}, g_{n+2}\right) \overline{\pi_{n}}\left(g_{n}\right) \overline{f_{\pi_{n}}}\left(\overline{g_{n}}\right) d g_{n}
$$

The integral $\Lambda\left(f_{0}, \overline{f_{\pi_{n}}}\right)$ converges for $\operatorname{Re}(s)>>0$, and we use analytic continuation to define $\Lambda\left(f_{0}, \overline{f_{\pi_{n}}}\right)$ elsewhere. Note that,

$$
\Lambda\left(f_{0}, \overline{f_{\pi_{n}}}\right) \in \operatorname{Ind}_{\mathrm{GL}\left(V^{\dagger} \cap Q\right) \times \overline{\mathrm{G}_{n}}}^{\overline{\mathrm{G}_{n+2}}} \Xi_{l} \otimes \chi_{0}^{-1 / 2} \otimes \overline{\pi_{n}}
$$

To see this, let $\left(a, \overline{g_{n}}\right) \in G L\left(V^{\dagger} \cap Q\right) \times \overline{G_{n}}$. Then,

$$
\Lambda\left(f_{0}, \overline{f_{\pi_{n}}}\right)\left(\left(a, \overline{g_{n}}\right) g_{n+2}\right)=\int_{\overline{\mathrm{G}_{n}}} \widetilde{f}_{0}\left(g_{n},\left(a, \overline{g_{n}}\right) g_{n+2}\right) \overline{\pi_{n}}\left(g_{n}\right) \overline{f_{\pi_{n}}} d g_{n}
$$




$$
\begin{aligned}
& =\int_{\overline{\mathrm{G}_{n}}} \widetilde{f}_{0}\left(g_{n},(a, 1)\left(1, \overline{g_{n}}\right) g_{n+2}\right) \overline{\pi_{n}}\left(g_{n}\right) \overline{f_{\pi_{n}}} d g_{n} \\
& (a, 1) \in \mathrm{GL}\left(V^{\dagger} \cap Q\right) \subseteq \mathrm{GL}\left(V^{\dagger}\right) \text { and } \operatorname{det}_{V^{\dagger}}(a, 1)=a \\
& =\Xi_{l} \chi_{0}^{-1 / 2}(a) \int_{\overline{\mathrm{G}_{n}}} \widetilde{f}_{0}\left(g_{n},\left(1, \overline{g_{n}}\right) g_{n+2}\right) \overline{\pi_{n}}\left(g_{n}\right) \overline{f_{\pi_{n}}} d g_{n} \\
& =\Xi_{l} \chi_{0}^{-1 / 2}(a) \int_{\overline{\mathrm{G}_{n}}} \tilde{f}_{0}\left(g_{n}, \overline{g_{n}} g_{n+2}\right) \overline{\pi_{n}}\left(g_{n}\right) \overline{f_{\pi_{n}}} d g_{n} \\
& =\Xi_{l} \chi_{0}^{-1 / 2}(a) \int_{\overline{\mathrm{G}_{n}}} \tilde{f}_{o}\left(\left(\overline{g_{n}}\right)^{-1} g_{n}, g_{n+2}\right) \overline{\pi_{n}}\left(g_{n}\right) \overline{f_{\pi_{n}}} d g_{n} \\
& =\Xi_{l} \chi_{0}^{-1 / 2}(a) \int_{\overline{\mathrm{G}_{n}}} \tilde{f}_{0}\left(g_{n}, g_{n+2}\right) \overline{\pi_{n}}\left(\overline{g_{n}} g_{n}\right) \overline{f_{\pi_{n}}} d g_{n} \\
& =\Xi_{l} \chi_{0}^{-1 / 2}(a) \overline{\pi_{n}}\left(\overline{g_{n}}\right) \int_{\overline{\mathrm{G}_{n}}} \tilde{f}_{0}\left(g_{n}, g_{n+2}\right) \overline{\pi_{n}}\left(g_{n}\right) \overline{f_{\pi_{n}}} d g_{n} \\
& =\Xi_{l} \chi_{0}^{-1 / 2}(a) \overline{\pi_{n}}\left(\overline{g_{n}}\right) \Lambda\left(f_{0}, \overline{f_{\pi_{n}}}\right)\left(g_{n+2}\right) .
\end{aligned}
$$

By the transitive property of induction, $\Lambda\left(f_{0}, \overline{f_{\pi_{n}}}\right)$ may be viewed as an element of $\overline{\pi_{n+2}}$.

Proposition 3.7. For any $k$ in $K_{n+2}, n$ a positive integer, and $\Lambda\left(f_{0}, \overline{f_{\pi_{n}}}\right)$ defined as above, we have

$$
\Lambda\left(f_{0}, \overline{f_{\pi_{n}}}\right)(k)=\frac{L\left(1, \overline{\pi_{n}} \otimes \Xi_{l} \otimes \chi_{0}^{-1 / 2}\right)}{\prod_{j=1}^{(n-1) / 2} L\left(1+2 j, \Xi_{l}^{2} \otimes \chi_{0}^{-1}\right)} \quad \text { for odd } n
$$

and

$$
\Lambda\left(f_{0}, \overline{f_{\pi_{n}}}\right)(k)=\frac{L\left(1, \overline{\pi_{n}} \otimes \Xi_{l} \otimes \chi_{0}^{-1 / 2}\right)}{\prod_{j=1}^{n / 2} L\left(2 j, \Xi_{l}^{2} \otimes \chi_{0}^{-1}\right)} \quad \text { for even } n
$$

Proof. Recall,

$$
\Lambda\left(f_{0}, \overline{f_{\pi_{n}}}\right)\left(k_{n+2}\right):=\int_{\overline{\mathrm{G}_{n}}} \hat{f}_{0}\left(g_{n}, k_{n+2}\right) \overline{\pi_{n}}\left(g_{n}\right) \overline{f_{\pi_{n}}} d g_{n} .
$$

Since $f_{0}$ is a spherical vector, $\Lambda$ is constant on $K_{n+2}$. Denote

$$
\mathrm{G}^{\square}=\mathrm{SO}\left(V \oplus V^{-}\right)
$$


which is isomorphic to $\mathrm{SO}_{2 n}$. Define $\hat{f}_{0}$ to be $f_{0}$ restricted to $\mathrm{G}^{\square}$. In viewing $f_{0} \in I\left(\Xi_{l}\right)$, then $f_{0}$ is a function on $\mathrm{SO}(W)$ to the complex numbers such that

$$
f_{0}(p g)=\Xi_{l} \chi_{0}^{-1 / 2} \circ \operatorname{det}_{V^{\dagger}}(m) \delta_{P}^{1 / 2} f_{0}(g)
$$

Let, $p \in P_{\mathrm{G}^{\square}} \cap \mathrm{G}^{\square}$ and let $g \in \mathrm{G}^{\square}$. Then,

$$
\begin{aligned}
\hat{f}_{0}(p g) & =\Xi_{l} \chi_{0}^{-1 / 2} \circ \operatorname{det}_{V^{\dagger}}(p) \delta_{P}^{1 / 2}(p) \hat{f}_{0}(g) \\
& =\Xi_{l} \chi_{0}^{-1 / 2} \circ \operatorname{det}_{V^{\dagger}}(p)\left(\frac{\delta_{P}}{\delta_{P} \cap \mathrm{G}^{\square}}\right)^{1 / 2}(p) \hat{f}_{0}(g)\left(\delta_{P} \cap \mathrm{G}^{\square}\right)^{1 / 2} .
\end{aligned}
$$

The induction is normalized, so

$$
\hat{f}_{0} \in \operatorname{Ind}_{P_{\mathrm{G}} \mathrm{G}^{\square} \cap \mathrm{G}^{\square}}\left(\frac{\delta_{P}}{\delta_{P} \cap \mathrm{G}^{\square}}\right)^{1 / 2} \Xi_{l} \otimes \chi_{0}^{-1 / 2} \circ \operatorname{det}_{V^{\dagger}} .
$$

We know that

$$
\delta_{P}(p)=\left|\operatorname{det}_{V^{\dagger}}(p)\right|^{n}
$$

To calculate $\delta_{P \cap G^{\square}}(p)$, note that $P=\operatorname{Stab}\left(V^{\dagger}\right)=\operatorname{Span}\left\{v_{i} \oplus-v_{i}, e\right\}$. Hence, $P \cap G^{\square}=$ $\operatorname{Stab}\left(\operatorname{Span}\left\{v_{i} \oplus-v_{i}\right\}\right)=P_{\mathrm{G}^{\square}}$. Hence,

$$
\delta_{P \cap G^{\square}}(p)=\left|\operatorname{det}_{V^{\dagger}}(p)\right|^{n-1} .
$$

Using Proposition 3 from [LR05], and noting that

$$
\Lambda\left(f_{0}, \overline{f_{\pi_{n}}}\right)(1)=\Lambda\left(\hat{f}_{0}, \overline{f_{\pi_{n}}}\right)(1)
$$

the proposition follows.

The previous proposition gives the following inductive relationship between $\zeta(\Xi, \xi)$ and $\zeta(\xi, \widetilde{\Xi})$. 
Proposition 3.8. For $n \geq 1$, we have

$$
\zeta(\Xi, \xi)=\zeta(\xi, \widetilde{\Xi}) \frac{L\left(1 / 2, \overline{\pi_{n+1}} \otimes \Xi_{l} \otimes \chi_{0}^{-1 / 2}\right)}{L\left(1, \overline{\pi_{n}} \otimes \Xi_{l} \otimes \chi_{0}^{-1 / 2}\right)} \times \begin{cases}L\left(1, \Xi_{l}^{2} \otimes \chi_{0}^{-1}\right)^{-1} & \text { if } n \text { is odd } \\ 1 & \text { if } n \text { is even } .\end{cases}
$$

Proof. We consider the integral

$$
\int_{\overline{G_{n+1}}} \hat{f}_{0}\left(1, g_{n+1}\right) \chi_{n+1}^{-1}\left(\nu\left(g_{n}^{-1} g_{n+1}\right)\right) \int_{\bar{G}_{n}} f_{\pi_{n+1}}\left(\eta_{n+1} g_{n} g_{n+1}\right) f_{\pi_{n}}\left(g_{n}\right) \omega^{-1}\left(\nu\left(g_{n}\right)\right) d g_{n} d g_{n+1} \text {. }
$$

We will utilize Proposition 3 from [LR05] again, but they consider an integral over $\mathrm{G}^{\diamond} \backslash(\mathrm{G} \times \mathrm{G})$ where $\mathrm{G}^{\diamond}=\{(g, g) \in \mathrm{G} \times \mathrm{G}\}$. The map between the sets $\mathrm{G}^{\diamond} \backslash(\mathrm{G} \times \mathrm{G}) \rightarrow$ G defined by $\left(g_{1}, g_{2}\right) \mapsto g_{1} g_{2}^{-1}$ is both injective and surjective. We use this isomorphism to consider the above integral instead. To see this, we follow the proof of R.N. Harris in [Har14], modifying to our case, and consider the pairing $\mathcal{T}: \pi_{n+1} \otimes \pi_{n} \rightarrow \mathbb{C}$ given by

$$
\mathcal{T}\left(f_{1}, f_{2}\right):=\int_{\overline{G_{n}}} f_{1}\left(\eta_{n+1} g_{n}\right) f_{2}\left(g_{n}\right) \omega^{-1}\left(\nu\left(g_{n+1} g_{n}\right)\right) d g_{n}
$$

Recall that $T_{\Xi, \xi}=\zeta(\Xi, \xi)$, so $\mathcal{T}\left(f_{\pi_{n+1}}, f_{\pi_{n}}\right)=\zeta(\xi, \tilde{\Xi})$. Also, note that $g_{n+1} \mapsto \tilde{f}_{0}\left(1, g_{n+1}\right)$ is bi-invariant under $K_{n+1}$. Choosing the Haar measure so that the volume on $K_{n+1}$ is 1 , we have

$$
\begin{aligned}
\int_{\overline{G_{n+1}}} \tilde{f}_{0}\left(1, g_{n+1}\right) \chi_{n+1}^{-1}\left(\nu\left(g_{n}^{-1} g_{n+1}\right)\right) \mathcal{T}\left(g_{n+1} f_{\pi_{n+1}}, f_{\pi_{n}}\right) d g_{n+1} \\
\quad=\int_{\overline{G_{n+1}}} \int_{K_{n+1}} \tilde{f}_{0}\left(1, k g_{n+1}\right) \chi_{n+1}^{-1}\left(\nu\left(k g_{n}^{-1} g_{n+1}\right)\right) \mathcal{T}\left(g_{n+1} f_{\pi_{n+1}}, f_{\pi_{n}}\right) d k d g_{n+1} \\
=\int_{\overline{G_{n+1}}} \int_{K_{n+1}} \tilde{f}_{0}\left(1, g_{n+1}\right) \chi_{n+1}^{-1}\left(\nu\left(g_{n}^{-1} g_{n+1}\right) \mathcal{T}\left(k^{-1} g_{n+1} f_{\pi_{n+1}}, f_{\pi_{n}}\right) d k d g_{n+1}\right. \\
=\int_{\overline{G_{n+1}}} \tilde{f}_{0}\left(1, g_{n+1}\right) \chi_{n+1}^{-1}\left(\nu\left(g_{n}^{-1} g_{n+1}\right)\right) \int_{K_{n+1}} \mathcal{T}\left(k^{-1} g_{n+1} f_{\pi_{n+1}}, f_{\pi_{n}}\right) d k d g_{n+1}
\end{aligned}
$$

The inner integral gives a smooth linear form on $\pi_{n+1}$. More specifically,

$$
f \mapsto \int_{K_{n+1}} \mathcal{T}\left(k^{-1} f, f_{\pi_{n}}\right)
$$


gives a map $L \in \pi_{n+1}^{\vee}$. Indeed, $L\left(g_{n+1} f_{\pi_{n+1}}\right)$ gives a matrix coefficient on $\pi_{n+1}$ which is bi-invariant under $K_{n+1}$. Thus, there is a constant $A$ such that

$$
L\left(g_{n+1} f_{\pi_{n+1}}\right)=A \cdot \mathcal{B}_{\pi_{n+1}}\left(g_{n+1} f_{\pi_{n+1}}, f_{\pi_{n+1}}\right) .
$$

To compute $A$, take $g_{n+1}=1$ and we obtain $L\left(f_{\pi_{n+1}}\right)=A$ so $L\left(f_{\pi_{n+1}}\right)=\mathcal{T}\left(f_{\pi_{n+1}}, f_{\pi_{n}}\right)$ and hence $\zeta(\xi, \tilde{\Xi})=A$. Now using the result of [LR05], we have

$$
\begin{aligned}
\int_{\overline{G_{n+1}}} \hat{f}_{0}\left(1, g_{n+1}\right) \chi_{n+1}^{-1}\left(\nu\left(g_{n}^{-1} g_{n+1}\right)\right) \int_{\overline{G_{n}}} f_{\pi_{n+1}}\left(\eta_{n+1} g_{n} g_{n+1}\right) f_{\pi_{n}}\left(g_{n}\right) \omega^{-1}\left(\nu\left(g_{n}\right)\right) d g_{n} d g_{n+1} \\
=\frac{L\left(1 / 2, \overline{\pi_{n+1}} \otimes \Xi_{l} \otimes \chi_{0}^{-1 / 2}\right)}{(n+1) / 2} \zeta(\xi, \widetilde{\Xi}) \\
\prod_{j=1} L\left(2 j-1, \Xi_{l}^{2} \otimes \chi_{0}^{-1}\right)
\end{aligned}
$$

for $n$ odd, and

$$
\begin{gathered}
\int_{\overline{G_{n+1}}} \hat{f}_{0}\left(1, g_{n+1}\right) \chi_{n+1}^{-1}\left(\nu\left(g_{n}^{-1} g_{n+1}\right)\right) \int_{\overline{G_{n}}} f_{\pi_{n+1}}\left(\eta_{n+1} g_{n} g_{n+1}\right) f_{\pi_{n}}\left(g_{n}\right) \omega^{-1}\left(\nu\left(g_{n}\right)\right) d g_{n} d g_{n+1} \\
=\frac{L\left(1 / 2, \overline{\pi_{n+1}} \otimes \Xi_{l} \otimes \chi_{0}^{-1 / 2}\right)}{\prod_{j=1}^{n / 2} L\left(2 j, \Xi_{l}^{2} \otimes \chi_{0}^{-1}\right)} \zeta(\xi, \widetilde{\Xi})
\end{gathered}
$$

for $n$ even. Using Proposition 3.8, we have

$$
\begin{gathered}
\int_{\overline{G_{n+1}}} \hat{f}_{0}\left(1, g_{n+1}\right) \chi_{n+1}^{-1}\left(\nu\left(g_{n}^{-1} g_{n+1}\right)\right) \int_{\overline{G_{n}}} f_{\pi_{n+1}}\left(\eta_{n+1} g_{n} g_{n+1}\right) f_{\pi_{n}}\left(g_{n}\right) \\
\cdot \omega^{-1}\left(\nu\left(g_{n}\right)\right) d g_{n} d g_{n+1} \\
=\int_{\overline{G_{n+1}}} \int_{\overline{G_{n}}} f_{\pi_{n+1}}\left(\eta_{n+1} g_{n} g_{n+1}\right) \hat{f}_{0}\left(g_{n}, g_{n} g_{n+1}\right) f_{\pi_{n}}\left(g_{n}\right) \\
\cdot \omega^{-1}\left(\nu\left(g_{n}\right)\right) \chi_{n+1}^{-1}\left(\nu\left(g_{n+1}\right)\right) d g_{n} d g_{n+1} \\
=\int_{\overline{G_{n}}} \int_{\overline{G_{n}}} f_{\pi_{n+1}}\left(\eta_{n+1} g_{n+1}\right) \hat{f}_{0}\left(g_{n}, g_{n+1}\right) f_{\pi_{n}}\left(g_{n}\right) \omega^{-1}\left(\nu\left(g_{n}\right)\right) \\
\cdot \chi_{n+1}^{-1}\left(\nu\left(g_{n}^{-1} g_{n+1}\right)\right) d g_{n} d g_{n+1}
\end{gathered}
$$




$$
\begin{aligned}
& =\int_{\overline{G_{n+1}}} \int_{\overline{G_{n}}} f_{\pi_{n+1}}\left(\eta_{n+1} g_{n+1}\right) \hat{f}_{0}\left(g_{n}, g_{n+1}\right) f_{\pi_{n}}\left(g_{n}\right) \omega^{-1}\left(\nu\left(g_{n}\right)\right) \chi_{n+1}\left(\nu\left(g_{n}\right)\right) \\
& \cdot \chi_{n+1}^{-1}\left(\nu\left(g_{n+1}\right)\right) d g_{n} d g_{n+1} \\
& =\int_{\overline{G_{n+1}}} f_{\pi_{n+1}}\left(\eta_{n+1} g_{n+1}\right) \chi_{n+1}^{-1}\left(\nu\left(g_{n+1}\right)\right) \int_{\overline{G_{n}}} \hat{f}_{0}\left(g_{n}, g_{n+1}\right) \pi_{n}(1) f_{\pi_{n}}\left(g_{n}\right) \\
& \omega^{-1}\left(\nu\left(g_{n}\right)\right) \chi_{n+1}\left(\nu\left(g_{n}\right)\right) d g_{n+1} d g_{n} \\
& =\int_{\overline{G_{n+1}}} f_{\pi_{n+1}}\left(\eta_{n+1} g_{n+1}\right) \chi_{n+1}^{-1}\left(\nu\left(g_{n+1}\right)\right) \int_{\overline{G_{n}}} \hat{f}_{0}\left(g_{n}, g_{n+1}\right) \pi_{n}(1) \overline{f_{\pi_{n}}}\left(g_{n}\right) d g_{n} d g_{n+1} \\
& =\int_{\overline{G_{n+1}}} f_{\pi_{n+1}}\left(\eta_{n+1} g_{n+1}\right) \chi_{n+1}^{-1}\left(\nu\left(g_{n+1}\right)\right) \Lambda\left(\hat{f}_{0}, \overline{f_{\pi_{n}}}\right)\left(g_{n+1}\right)(1) d g_{n+1} \\
& =\frac{L\left(1, \overline{\pi_{n}} \otimes \Xi_{l} \otimes \chi_{0}^{-1 / 2}\right)}{(n-1) / 2} \int_{\overline{G_{n+1}}} f_{\pi_{n+1}}\left(\eta_{n+1} g_{n+1}\right) \chi_{n+1}^{-1}\left(\nu\left(g_{n+1}\right)\right) \\
& \prod_{j=1} L\left(1+2 j, \Xi_{l}^{2} \otimes \chi_{0}^{-1}\right) \\
& =\frac{L\left(1, \overline{\pi_{n}} \otimes \Xi_{l} \otimes \chi_{0}^{-1 / 2}\right)}{(n-1) / 2} \int_{\overline{G_{n+1}}} f_{\pi_{n+1}}\left(\eta_{n+1} g_{n+1}\right) f_{\pi_{n+2}}\left(g_{n+1}\right) \chi_{n+1}\left(\nu\left(g_{n+1}\right)\right. \\
& \prod_{j=1} L\left(1+2 j,\left(\Xi_{l}^{2} \otimes \chi_{0}^{-1}\right)\right. \\
& \cdot \omega^{-1}\left(\nu\left(g_{n+1}\right)\right) \chi_{n+1}^{-1}\left(\nu\left(g_{n+1}\right)\right) d g_{n+1} \\
& =\frac{L\left(1, \overline{\pi_{n}} \otimes \Xi_{l} \otimes \chi_{0}^{-1 / 2}\right)}{\prod_{j=1}^{(n-1) / 2} L\left(1+2 j, \Xi_{l}^{2} \otimes \chi_{0}^{-1 / 2}\right)} \int_{\overline{G_{n+1}}} f_{\pi_{n+1}}\left(g_{n+1}\right) f_{\pi_{n+2}}\left(\eta_{n+1}^{-1} g_{n+1}\right) \\
& \cdot \omega^{-1}\left(\nu\left(\eta_{n+1}^{-1} g_{n+1}\right)\right) d g_{n+1} \\
& =\frac{L\left(1, \overline{\pi_{n}} \otimes \Xi_{l} \otimes \chi_{0}^{-1 / 2}\right)}{(n-1) / 2} T_{\Xi, \xi}\left(\eta_{n+1}^{-1}\right)=\frac{L\left(1, \overline{\pi_{n}} \otimes \Xi_{l} \otimes \chi_{0}^{-1 / 2}\right)}{(n-1) / 2} \zeta(\Xi, \xi) \\
& \prod_{j=1} L\left(1+2 j, \Xi_{l}^{2} \otimes \chi_{0}^{-1}\right) \quad \prod_{j=1} L\left(1+2 j, \Xi_{l}^{2} \otimes \chi_{0}^{-1}\right)
\end{aligned}
$$

for $n$ odd. Since $T_{\Xi, \xi}$ is independent of the choice of $\eta, T_{\Xi \xi}\left(\eta_{n+1}^{-1}\right)=\zeta(\Xi, \xi)$. Putting these results together, we have

$$
\frac{L\left(1 / 2, \overline{\pi_{n+1}} \otimes \Xi_{l} \otimes \chi_{0}^{-1 / 2}\right)}{\prod_{j=1}^{(n+1) / 2} L\left(2 j-1, \Xi_{l}^{2} \otimes \chi_{0}^{-1}\right)} \zeta(\xi, \widetilde{\Xi})=\frac{L\left(1, \overline{\pi_{n}} \otimes \Xi_{l} \otimes \chi_{0}^{-1 / 2}\right)}{\prod_{j=1}^{(n-1) / 2} L\left(1+2 j, \Xi_{l}^{2} \otimes \chi_{0}^{-1}\right)} \zeta(\Xi, \xi) .
$$

Solving for $\zeta(\Xi, \xi)$ completes the proof for $n$ odd. 
Similarly for $n$ even, we have

$$
\frac{L\left(1 / 2, \overline{\pi_{n+1}} \otimes \Xi_{l} \otimes \chi_{0}^{-1 / 2}\right)}{\prod_{j=1}^{n / 2} L\left(2 j, \Xi_{l}^{2} \otimes \chi_{0}^{-1}\right)} \zeta(\xi, \widetilde{\Xi})=\frac{L\left(1, \bar{\pi}_{n} \otimes \Xi_{l} \otimes \chi_{0}^{-1 / 2}\right)}{\prod_{j=1}^{n / 2} L\left(2 j, \Xi_{l}^{2} \otimes \chi_{0}^{-1}\right)} \zeta(\Xi, \xi) .
$$

Solving for $\zeta(\Xi, \xi)$, the proposition follows.

By induction, we have the following Corollary.

Corollary 3.9. Suppose $E=F$. If $n$ is odd, then

$$
\begin{gathered}
\zeta(\Xi, \xi)=\prod_{i=1}^{(n+1) / 2} L\left(1, \Xi_{i}^{2} \chi_{0}^{-1}\right)^{-1} \prod_{1 \leq i<j \leq(n+1) / 2} L\left(1, \Xi_{i} \Xi_{j} \chi_{0}^{-1}\right)^{-1} L\left(1, \Xi_{i}^{-1} \Xi_{j}\right)^{-1} \\
\times \prod_{1 \leq i<j \leq(n+1) / 2} L\left(1, \xi_{i} \xi_{j} \chi_{1}^{-1}\right)^{-1} L\left(1, \xi_{i}^{-1} \xi_{j}\right)^{-1} \\
\times \prod_{1 \leq i<j \leq(n+1) / 2} L\left(1 / 2, \Xi_{i} \xi_{j} \chi_{0}^{-1 / 2} \chi_{1}^{-1 / 2}\right) L\left(1 / 2, \Xi_{i}^{-1} \xi_{j} \chi_{0}^{1 / 2} \chi_{1}^{-1 / 2}\right) \\
\times \prod_{1 \leq j \leq i \leq(n+1) / 2} L\left(1 / 2, \Xi_{i} \xi_{j} \chi_{0}^{-1 / 2} \chi_{1}^{-1 / 2}\right) L\left(1 / 2, \Xi_{i} \xi_{j}^{-1} \chi_{0}^{-1 / 2} \chi_{1}^{1 / 2}\right) .
\end{gathered}
$$

If $n$ is even, then

$$
\begin{gathered}
\zeta(\Xi, \xi)=\prod_{1 \leq i<j \leq n / 2+1} L\left(1, \Xi_{i} \Xi_{j} \chi_{0}^{-1}\right)^{-1} L\left(1, \Xi_{i}^{-1} \Xi_{j}\right)^{-1} \\
\times \prod_{i=1}^{n / 2} L\left(1, \xi_{i}^{2} \chi_{1}^{-1}\right)^{-1} \prod_{1 \leq i<j \leq n / 2} L\left(1, \xi_{i} \xi_{j} \chi_{1}^{-1}\right)^{-1} L\left(1, \xi_{i}^{-1} \xi_{j}\right)^{-1} \\
\times \prod_{1 \leq i<j \leq n / 2} L\left(1 / 2, \Xi_{i} \xi_{j} \chi_{0}^{-1 / 2} \chi_{1}^{-1 / 2}\right) L\left(1 / 2, \Xi_{i}^{-1} \xi_{j} \chi_{0}^{1 / 2} \chi_{1}^{-1 / 2}\right) \\
\times \prod_{1 \leq j \leq i \leq n / 2+1} L\left(1 / 2, \Xi_{i} \xi_{j} \chi_{0}^{-1 / 2} \chi_{1}^{-1 / 2}\right) L\left(1 / 2, \Xi_{i} \xi_{j}^{-1} \chi_{0}^{-1 / 2} \chi_{1}^{1 / 2}\right) .
\end{gathered}
$$

Proof. The base case deals with $\mathrm{G}_{1}$ and $\mathrm{G}_{1}=\mathrm{GSpin}_{1}=\mathrm{GL}(1)$. By Lemma 2.5 $A_{1}=G L(1)$, so $\overline{G_{1}}$ is trivial. The induction step follows from the previous proposition. 


\subsubsection{Calculation of $S_{\Xi^{-1}, \xi^{-1}}$ when $E=F$}

On the other hand,

$$
S_{\Xi^{-1}, \xi^{-1}}(1)=\int_{K_{n+2} \times K_{n+1}} Y_{\Xi, \xi}\left(k_{n+2} k_{n+1}\right) d k_{n+2} d k_{n+1}
$$

The similitude character is trivial on compact sets. Thus, we have the following commutative diagram:

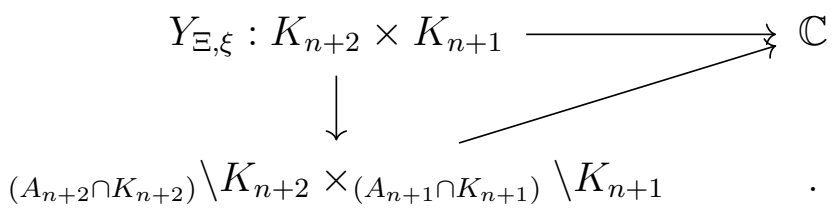

Hence, $Y_{\Xi, \xi}^{\mathrm{GSpin}}{ }_{m}$ restricted to $K_{n+2}^{\mathrm{GSpin}} \times K_{n+1}^{\mathrm{GSpin}}$ is isomorphic to $Y_{\Xi^{\prime}, \xi^{\prime}}^{\mathrm{SO}}$ restricted to $K_{n+2}^{\mathrm{SO}} \times K_{n+1}^{\mathrm{SO}}$. Here if $\xi=\left(\xi_{1}, \ldots, \xi_{\lfloor(n+1) / 2\rfloor}, \chi_{1}\right)$ and $\Xi=\left(\Xi_{1}, \ldots, \Xi_{\lfloor(n+2) / 2\rfloor}, \chi_{0}\right)$ are representations on GSpin, then $\xi^{\prime}:=\left(\xi_{1}, \ldots, \xi_{\lfloor(n+1) / 2\rfloor}\right)$ and $\Xi^{\prime}:=\left(\Xi_{1}, \ldots, \Xi_{\lfloor(n+2) / 2\rfloor}\right)$ are representations on $\mathrm{SO}$.

Since both $G_{n+2}$ and $G_{n+1}$ are split and there exists the above relationship with the special orthogonal groups, we use [KMS03, Thm 10.8] to give the following:

$$
\begin{aligned}
S_{\Xi^{-1}, \xi^{-1}}(1)= & \Delta_{G_{n+2}} \zeta(1)^{-(n+1)} \prod_{i=1}^{(n+1) / 2} L\left(1, \Xi_{i}^{-2} \chi_{0}\right)^{-1} \\
& \times \prod_{1 \leq i<j \leq(n+1) / 2} L\left(1, \Xi_{i}^{-1} \Xi_{j}^{-1} \chi_{0}\right) L\left(1, \Xi_{i} \Xi_{j}^{-1}\right)^{-1} \\
& \times \prod_{1 \leq i<j \leq(n+1) / 2} L\left(1, \xi_{i}^{-1} \xi_{j}^{-1} \chi_{1}\right)^{-1} L\left(1, \xi_{i} \xi_{j}^{-1}\right)^{-1} \\
& \times \prod_{1 \leq i<j \leq(n+1) / 2} L\left(1 / 2, \Xi_{i}^{-1} \xi_{j}^{-1} \chi_{0}^{1 / 2} \chi_{1}^{1 / 2}\right) L\left(1 / 2, \Xi_{i} \xi_{j}^{-1} \chi_{0}^{-1 / 2} \chi_{1}^{1 / 2}\right) \\
& \times \prod_{1 \leq j \leq i \leq(n+1) / 2} L\left(1 / 2, \Xi_{i}^{-1} \xi_{j}^{-1} \chi_{0}^{1 / 2} \chi_{1}^{1 / 2}\right) L\left(1 / 2, \Xi_{i}^{-1} \xi_{j} \chi_{0}^{1 / 2} \chi_{1}^{-1 / 2}\right)
\end{aligned}
$$

for $n$ odd, and

$$
S_{\Xi^{-1}, \xi^{-1}}(1)=\Delta_{G_{n+2}} \zeta(1)^{-(n+1)} \prod_{i=1}^{n / 2} L\left(1, \xi_{i}^{-2} \chi_{1}\right)^{-1}
$$




$$
\begin{aligned}
& \times \prod_{1 \leq i<j \leq n / 2+1} L\left(1, \Xi_{i}^{-1} \Xi_{j}^{-1} \chi_{0}\right) L\left(1, \Xi_{i} \Xi_{j}^{-1}\right)^{-1} \\
& \times \prod_{1 \leq i<j \leq n / 2} L\left(1, \xi_{i}^{-1} \xi_{j}^{-1} \chi_{1}\right)^{-1} L\left(1, \xi_{i} \xi_{j}^{-1}\right)^{-1} \\
& \times \prod_{1 \leq i<j \leq n / 2} L\left(1 / 2, \Xi_{i}^{-1} \xi_{j}^{-1} \chi_{0}^{1 / 2} \chi_{1}^{1 / 2}\right) L\left(1 / 2, \Xi_{i} \xi_{j}^{-1} \chi_{0}^{-1 / 2} \chi_{1}^{1 / 2}\right) \\
& \times \prod_{1 \leq j \leq i \leq n / 2+1} L\left(1 / 2, \Xi_{i}^{-1} \xi_{j}^{-1} \chi_{0}^{1 / 2} \chi_{1}^{1 / 2}\right) L\left(1 / 2, \Xi_{i}^{-1} \xi_{j} \chi_{0}^{1 / 2} \chi_{1}^{-1 / 2}\right)
\end{aligned}
$$

for $n$ even and

$$
\Delta_{G_{i}}= \begin{cases}\zeta(2) \zeta(4) \cdots \zeta(2 m) & \text { if } \operatorname{dim} V_{i}=2 m+1, \\ \zeta(2) \zeta(4) \cdots \zeta(2 m-2) & \text { if } \operatorname{dim} V_{i}=2 m\end{cases}
$$

If $n$ is odd, let $n+2=2 m+1$. From (2.3) we have

$$
\begin{aligned}
L\left(s, \pi_{n+2}, \text { Ad }\right) & =\left(\frac{1}{1-q^{-s}}\right)^{m} \prod_{i \neq j} \frac{1}{1-\Xi_{i}^{ \pm 1} \Xi_{j}^{\mp 1} q^{-s}} \cdot \frac{1}{1-\Xi_{i}^{ \pm 1} \Xi_{j}^{ \pm 1} \chi_{0}^{\mp 1} q^{-s}} \\
& \times \prod_{1 \leq i \leq(n+1) / 2} \frac{1}{1-\Xi_{i}^{2} \chi_{0}^{-1} q^{-s}} \cdot \frac{1}{1-\Xi_{i}^{-2} \chi_{0} q^{-s}} .
\end{aligned}
$$

Hence,

$$
\begin{aligned}
L\left(s, \pi_{n+2}, \mathrm{Ad}\right)= & \zeta(s)^{(m)} \prod_{1 \leq i, j \leq(n+1) / 2} L\left(s, \Xi_{i} \Xi_{j}^{-1}\right) L\left(s, \Xi_{i} \Xi_{j} \chi_{0}^{-1}\right) \\
& \times \prod_{1 \leq i, j \leq(n+1) / 2} L\left(s, \Xi_{i}^{-1} \Xi_{j}\right) L\left(s, \Xi_{i}^{-1} \Xi_{j}^{-1} \chi_{0}\right) \\
& \times \prod_{1 \leq i \leq(n+1) / 2} L\left(s, \Xi_{i}^{2} \chi_{0}^{-1}\right) L\left(s, \Xi_{i}^{-2} \chi_{0}\right) .
\end{aligned}
$$

For $n+1=2 m$, we use (2.4) and we have

$$
L\left(s, \pi_{n+1}, A d\right)=\left(\frac{1}{1-q^{-s}}\right)^{m} \prod_{i \neq j} \frac{1}{1-q^{-s} \xi_{i}^{ \pm 1} \xi_{j}^{\mp 1}} \cdot \frac{1}{1-q^{-s} \xi_{i}^{ \pm 1} \xi_{j}^{ \pm 1} \chi_{0}^{\mp 1}} .
$$

Hence,

$$
\begin{aligned}
L\left(s, \pi_{n+1}, \mathrm{Ad}\right)= & \zeta(s)^{(m)} \prod_{1 \leq i, j \leq(n+1) / 2} L\left(s, \xi_{i} \xi_{j}^{-1}\right) L\left(s, \xi_{i} \xi_{j} \chi_{1}^{-1}\right) \\
& \times \prod_{1 \leq i, j \leq(n+1) / 2} L\left(s, \xi_{i}^{-1} \xi_{j}\right) L\left(\xi_{i}^{-1} \xi_{j}^{-1} \chi_{1}\right) .
\end{aligned}
$$


Similarly for $n=2 m$, we have

$$
\begin{aligned}
L\left(s, \pi_{n+2}, \mathrm{Ad}\right)=\zeta(s)^{(m)} & \prod_{i \leq i<j \leq n / 2} L\left(s, \Xi_{i} \Xi_{j}^{-1}\right) L\left(s, \Xi_{i} \Xi_{j} \chi_{0}^{-1}\right) \\
& \times \prod_{i \leq i<j \leq n / 2} L\left(s, \Xi_{i}^{-1} \Xi_{j}\right) L\left(s, \Xi_{i}^{-1} \Xi_{j} \chi_{0}\right) .
\end{aligned}
$$

and

$$
\begin{aligned}
& L\left(s, \pi_{n+1}, \mathrm{Ad}\right)=\zeta(s)^{(m)} \prod_{\substack{1 \leq i, j \leq(n+1) / 2 \\
\times}} L\left(s, \xi_{i} \xi_{j}^{-1}\right) L\left(s, \xi_{i} \xi_{j} \chi_{1}^{-1}\right) \\
& \prod_{\substack{1 \leq i, j \leq(n+1) / 2\\
}} L\left(s, \xi_{i}^{-1} \xi_{j}\right) L\left(\xi_{i}^{-1} \xi_{j}^{-1} \chi_{1}\right) \\
& \prod_{1 \leq i \leq(n+1) / 2} L\left(s, \xi_{i}^{2} \chi_{1}^{-1}\right) L\left(s, \xi_{i}^{-2} \chi_{1}\right) .
\end{aligned}
$$

We also have,

$$
\begin{aligned}
L\left(s, \pi_{n+2} \times \pi_{n+1}\right)=\prod_{1 \leq i, j \leq\lfloor(n+1) / 2\rfloor} L\left(s, \Xi_{i} \xi_{j}\right) L\left(s, \Xi^{-1} \xi_{j} \chi_{0}\right) \\
\times L\left(s, \Xi_{i} \xi_{j}^{-1} \chi_{1}\right) L\left(\Xi_{i}^{-1} \xi_{j}^{-1} \chi_{0} \chi_{1}\right) .
\end{aligned}
$$

Tensoring by $\omega^{-1}$ gives

$$
\begin{aligned}
L\left(s, \pi_{n+2}\right. & \left.\otimes \omega^{-1} \times \pi_{n+1}\right)= \\
& \prod_{1 \leq i, j \leq\lfloor(n+1) / 2\rfloor} L\left(s, \Xi_{i} \xi_{j} \chi_{0}^{-1 / 2} \chi_{1}^{-1 / 2}\right) L\left(s, \Xi_{i} \xi_{j}^{-1} \chi_{0}^{-1 / 2} \chi_{1}^{1 / 2}\right) \\
\times & \prod_{1 \leq i, j \leq\lfloor(n+1) / 2\rfloor} L\left(s, \Xi_{i}^{-1} \xi_{j} \chi_{0}^{1 / 2} \chi_{1}^{-1 / 2}\right) L\left(s, \Xi_{i}^{-1} \xi_{j}^{-1} \chi_{0}^{1 / 2} \chi_{1}^{1 / 2}\right) .
\end{aligned}
$$

Thus, for each $v \notin S$,

$$
\begin{aligned}
\mathcal{P}^{\natural}\left(f_{\pi_{n+2}}, f_{\pi_{n+1}}\right) & =F(1)=\zeta(\Xi, \xi) S_{\Xi^{-1}, \xi^{-1}}(1) \\
& =\zeta(\Xi, \xi) \zeta\left(\Xi^{-1}, \xi^{-1}\right) \Delta_{G_{n+2}} \zeta(1)^{-(n+1)} . \\
& =\Delta_{G_{n+2}} \frac{L\left(1 / 2, \pi_{n+2} \otimes \omega^{-1} \times \pi_{n+1}\right)}{L\left(1, \pi_{n+2}, \mathrm{Ad}\right)\left(L\left(1, \pi_{n+1}, \mathrm{Ad}\right)\right.} .
\end{aligned}
$$

where

$$
\Delta_{\mathrm{G}_{i}}= \begin{cases}\zeta(2) \zeta(4) \cdots \zeta(2 m) & \text { if } \operatorname{dim} V_{i}=2 m+1 \\ \zeta(2) \zeta(4) \cdots \zeta(2 m-2) L(m, \mu) & \text { if } \operatorname{dim} V_{i}=2 m\end{cases}
$$




\subsubsection{Calculations when $E / F$ is an unramified quadratic ex- tension}

There are quasi-split non-split forms in the even case. Note that these do not exist in

the odd case. As in [AS14], fix a splitting $\left(B, T,\left\{x_{\alpha}\right\}_{\alpha \in \Delta}\right)$ where $\left\{x_{\alpha}\right\}$ is a collection of root vectors, one for each simple root of $T$ in $B$. The quasi-split forms of GSpin $(2 m)$ over $F$ are in one-to-one correspondence with homomorphisms from $\mathrm{Gal}(\bar{F} / F)$ to the group of automorphisms of the character lattice preserving $\Delta$. The only two elements in this group are the trivial element and the one switching the roots $e_{n-1}-e_{n}$ and $e_{n-1}+e_{n}$ while keeping all other simple roots fixed. As we had stated before, class field theory tells us such homomorphisms correspond to quadratic characters

$$
\mu: F^{\times} \rightarrow\{ \pm 1\}
$$

Note that

$$
\mathcal{A}_{n+2} \simeq\left(F^{\times}\right)^{\lfloor(n+1) / 2\rfloor}, \quad \mathcal{A}_{n+1} \simeq\left(F^{\times}\right)^{\lfloor n / 2\rfloor} .
$$

The unramified characters $\Xi$ and $\xi$ are determined by their restriction to their associated maximal split subtorus. Let

$$
\begin{gathered}
\Xi=\left(\Xi_{1}, \Xi_{2}, \ldots, \Xi_{\lfloor(n+1) / 2\rfloor}, \chi_{0}\right) \\
\xi=\left(\xi_{1}, \xi_{2}, \ldots, \xi_{\lfloor n / 2\rfloor}, \chi_{1}\right) .
\end{gathered}
$$

Put $\widetilde{\Xi}=\left(\Xi_{1}, \Xi_{2}, . . \Xi_{\lfloor(n-1) / 2\rfloor}, \chi_{0}\right)$. Set $\zeta(\Xi, \xi)=T_{\Xi, \xi}(\eta)$.

Hence we have the same result as before. Namely, for for a positive integer $n$,

$$
\zeta(\Xi, \xi)=\zeta(\xi, \widetilde{\Xi}) \frac{L\left(1 / 2, \overline{\pi_{n+1}} \otimes \Xi_{l} \otimes \chi_{0}^{-1 / 2}\right)}{L\left(1, \overline{\pi_{n}} \otimes \Xi_{l} \otimes \chi_{0}^{-1 / 2}\right)} \times \begin{cases}L\left(1, \Xi_{l}^{2} \otimes \chi_{0}^{-1}\right)^{-1} & \text { if } n \text { is odd } \\ 1 & \text { if } n \text { is even }\end{cases}
$$

Let $n=2 m$. Since $\pi_{n+2}$ is unramified and $\mathrm{G}_{n+2}$ is quasi-split, but not split, there is a one-to-one correspondence between $\pi_{n+2}$ and the Satake parameters for $\mathrm{G}_{n+2}$ given 
by [AS14, Equation 3.32]

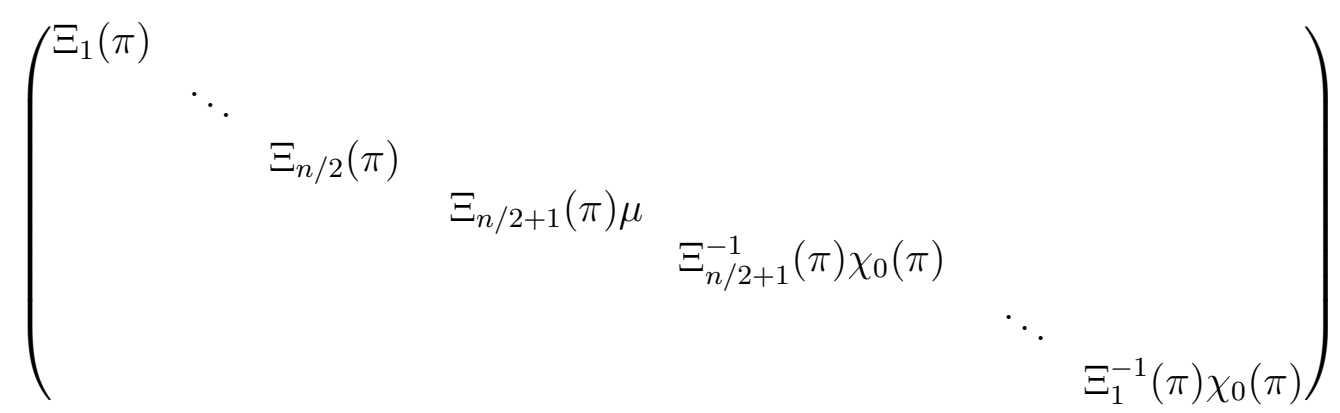

where $\mu$ is the quadratic character associated to $E / F$. By induction, when $n$ is odd we have,

$$
\begin{gathered}
\zeta(\Xi, \xi)=\prod_{i=1}^{(n+1) / 2} L\left(1, \Xi_{i}^{2} \chi_{0}^{-1}\right)^{-1} \prod_{1 \leq i<j \leq(n+1) / 2} L\left(1, \Xi_{i} \Xi_{j} \chi_{0}^{-1}\right)^{-1} L\left(1, \Xi_{i}^{-1} \Xi_{j}\right)^{-1} \\
\times \prod_{i=1}^{(n-1) / 2} L\left(1, \xi_{i} \chi_{1}^{-1 / 2}\right) L\left(1, \mu \xi_{i} \chi_{1}^{-1 / 2}\right) \times \prod_{1 \leq i<j \leq(n-1) / 2} L\left(1, \xi_{i} \xi_{j} \chi_{1}^{-1}\right)^{-1} L\left(1, \xi_{i}^{-1} \xi_{j}\right)^{-1} \\
\times \prod_{1 \leq i<j \leq(n-1) / 2} L\left(1 / 2, \Xi_{i} \xi_{j} \chi_{0}^{-1 / 2} \chi_{1}^{-1 / 2}\right) L\left(1 / 2, \Xi_{i}^{-1} \xi_{j} \chi_{0}^{1 / 2} \chi_{1}^{-1 / 2}\right) \\
\times \prod_{i=1}^{(n+1) / 2} L\left(1 / 2, \Xi_{i} \chi_{0}^{1 / 2}\right) L\left(1 / 2, \mu \Xi_{i} \chi_{0}^{1 / 2}\right) \\
\times \prod_{1 \leq j \leq i \leq(n+1) / 2} L\left(1 / 2, \Xi_{i} \xi_{j} \chi_{0}^{-1 / 2} \chi_{1}^{-1 / 2}\right) L\left(1 / 2, \Xi_{i} \xi_{j}^{-1} \chi_{0}^{-1 / 2} \chi_{1}^{1 / 2}\right) .
\end{gathered}
$$

When $n$ is even,

$$
\begin{aligned}
\zeta(\Xi, \xi)= & \prod_{i=1}^{n / 2} L\left(1, \Xi_{i} \chi_{0}^{1 / 2}\right)^{-1} L\left(1, \mu \Xi_{i} \chi_{0}^{1 / 2}\right)^{-1} \\
& \prod_{1 \leq i<j \leq n / 2} L\left(1, \Xi_{i} \Xi_{j} \chi_{0}^{-1}\right)^{-1} L\left(1, \Xi_{i}^{-1} \Xi_{j}\right)^{-1} \\
& \times \prod_{i=1}^{n / 2} L\left(1, \xi_{i}^{2} \chi_{1}^{-1}\right)^{-1} \prod_{1 \leq i<j \leq n / 2} L\left(1, \xi_{i} \xi_{j} \chi_{1}^{-1}\right)^{-1} L\left(1, \xi_{i}^{-1} \xi_{j}\right)^{-1} \\
& \prod_{1 \leq j \leq i \leq n / 2} L\left(1 / 2, \Xi_{i} \xi_{j} \chi_{0}^{-1 / 2} \chi_{1}^{-1 / 2}\right) L\left(1 / 2, \Xi_{i} \xi_{j}^{-1} \chi_{0}^{-1 / 2} \chi_{1}^{1 / 2}\right) \\
& \times \prod_{1 \leq i<j \leq n / 2} L\left(1 / 2, \Xi_{i} \xi_{j} \chi_{0}^{-1 / 2} \chi_{1}^{-1 / 2}\right) L\left(1 / 2, \Xi_{i}^{-1} \xi_{j} \chi_{0}^{1 / 2} \chi_{1}^{-1 / 2}\right) \\
& \times \prod_{i=1}^{n / 2} L\left(1 / 2, \xi_{i}\right) L\left(1 / 2, \mu \xi_{i}\right) .
\end{aligned}
$$


Using Lemma 5.1 in [II10], we have that

$$
S_{\Xi, \xi}(1)=\Delta_{G_{n+2}} \zeta(1)^{-\operatorname{dim} \mathcal{A}_{n+2}-\operatorname{dim} \mathcal{A}_{n+1}} L(1, \mu)^{-1} \zeta(\Xi, \xi)
$$

Note that,

$$
L_{\pi_{n+2}, \pi_{n+1}}(1 / 2)=\zeta(1)^{-\operatorname{dim}\left(\mathcal{A}_{n+2}+1\right)-\operatorname{dim}\left(\mathcal{A}_{n+1}+1\right)} L(1, \mu)^{-1} \zeta(\Xi, \xi) \zeta\left(\Xi^{-1}, \xi^{-1}\right) .
$$

Using the results from the previous sections, we have the following theorem.

Theorem 3.10. For each $v \notin S$

$$
\begin{aligned}
\mathcal{P}^{\natural}\left(f_{\pi_{n+2}}, f_{\pi_{n+1}}\right) & =F(1)=\zeta(\Xi, \xi) S_{\Xi^{-1}, \xi^{-1}}(1) \\
& =\Delta_{G_{n+2}} \frac{L\left(1 / 2, \pi_{n+2} \otimes \omega^{-1} \times \pi_{n+1}\right)}{L\left(1, \pi_{n+2}, \mathrm{Ad}\right) L\left(1, \pi_{n+1}, \mathrm{Ad}\right)} .
\end{aligned}
$$




\section{Chapter 4}

\section{The Global Gan-Gross-Prasad Conjecture for $\mathrm{GSpin}_{2} \times \mathrm{GSpin}_{3}$}

Let $F$ be an algebraic number field with adele ring $\mathbb{A}_{F}$. Denote by $D$ the quaternion algebra over $F$ and define $E$ as a quadratic field extension of $F$ with a fixed embedding $E \hookrightarrow D$ over $F$. We consider the following exceptional isomorphisms of GSpin [AS14, section 4.1]:

$$
\mathrm{GSpin}_{2}= \begin{cases}\operatorname{Res}_{E / F} \mathrm{GL}_{1} & \text { if quasi-split } \\ \mathrm{GL}_{1} \times \mathrm{GL}_{1} & \text { if split }\end{cases}
$$

and

$$
\mathrm{GSpin}_{3}= \begin{cases}\mathrm{GL}_{2} & \text { if } D=M_{2}(F) \\ D^{\times} & \text {if } D \text { is non-split. }\end{cases}
$$

We use the Waldspurger formula [Wal85] to prove the cases where GSpin ${ }_{2}$ is $\operatorname{Res}_{E / F} \mathrm{GL}_{1}$ and $D$ is either split or non-split. The case where $\mathrm{GL}_{1} \times \mathrm{GL}_{1} \subseteq D^{\times}$does not occur. When GSpin ${ }_{2}$ is split, there is an embedding $\mathrm{GL}_{1} \times \mathrm{GL}_{1} \subseteq \mathrm{GL}_{2}$. We work through this case, but it does not confirm Conjecture 1.3 or 1.4.

\subsection{Waldspurger Formula}

The following example is due to Waldspurger [Wal85]. First, we give a brief discussion of the Waldspurger formula ([Wal85]). Another good source for the Waldspurger formula is [YZZ13, Ch. 1]. 


\subsubsection{Linear forms over local fields}

Let $F$ be a local field and $D$ a quaternion algebra over $F$. Then $D$ is either isomorphic to $M_{2}(F)$ or the unique division quaternion algebra over $F$. Let $E$ be either $F \oplus F$ or a quadratic field extension over $F$, with a fixed embedding $E \hookrightarrow D$ of algebras over $F$. Let $\mu$ be the quadratic character associated to $E / F$ by class field theory. Let $\pi$ be an irreducible cuspidal representation of $D^{\times}$with central character $\omega_{\pi}$, and let $\chi: E^{\times} \rightarrow \mathbb{C}$ be a character on $E^{\times}$such that $\omega_{\pi} \cdot \chi$ restricted to $F^{\times}$is trivial.

Fix a Haar measure $d t$ on $E^{\times} / F^{\times}$. Define a bilinear form

$$
\alpha: \pi \otimes \tilde{\pi} \rightarrow \mathbb{C}
$$

by

$$
\alpha\left(f_{1}, f_{2}\right)=\frac{L(1, \pi, \mathrm{Ad}) L(1, \mu)}{\zeta(2) L(1 / 2, \pi \otimes \chi)} \int_{F^{\times} \backslash E^{\times}} \mathcal{B}_{\pi}\left(\pi(t) f_{1}, f_{2}\right) d t, \quad f_{1} \in \pi, f_{2} \in \tilde{\pi} .
$$

where $\mathcal{B}_{\pi}\left(\pi(t) f_{1}, f_{2}\right)$ is the fixed inner product discussed in chapter one.

\subsubsection{Waldspurger formula}

Now let $F$ be a number field with adele ring $\mathbb{A}_{F}$. Denote by $D$ the quaternion algebra over $F$ and define $E$ as a quadratic field extension of $F$ with fixed embedding $E \hookrightarrow D$

over $F$. Let $\mu: \mathbb{A}_{F}^{\times} / F^{\times} \rightarrow \mathbb{C}$ be the quadratic character associated to the extension $E / F$. Let $\pi$ be an irreducible cuspidal automorphic representation of $D^{\times}$with central character $\omega_{\pi}$ and $\chi$ a Hecke character on $\mathbb{A}_{E}^{\times} / E^{\times}$. Define the period integral

$$
\widetilde{P_{\chi}}(f)=\left|\int_{E^{\times} \backslash \mathbb{A}_{E}^{\times} / \mathbb{A}^{\times}} f(t) \chi(t) d t\right|^{2}
$$

where $d t$ is the Tamagawa measure, which has total volume 2 on $E^{\times} \backslash \mathbb{A}_{E}^{\times} / \mathbb{A}^{\times}$. Let $\mathcal{B}_{\pi_{v}}$ and $\mathcal{B}_{\chi_{v}}$ be local pairings. We choose local measures $d t_{v}$ so that $d t=\prod_{v} d t_{v}$. Let 
$\mathcal{B}(\cdot, \cdot)$ be the Petersson inner product on $\pi$. That is,

$$
\mathcal{B}_{\pi}\left(\pi(t) f_{1}, f_{2}\right)=\int_{D^{\times} \backslash D\left(\mathbb{A}^{\times}\right) / \mathbb{A}^{\times}} f_{1}(b) \overline{f_{2}(b)} d b, \quad f_{1} \in \pi, f_{2} \in \tilde{\pi}
$$

where $d b$ is the Tamagawa measure. Let $B C(\pi)$ denote the base change of $\pi$ to $E$.

Theorem 4.1. (Waldspurger [Wal85]) For any $f_{1} \in \pi$ and $\overline{f_{1}} \in \bar{\pi}$, we have

$$
\widetilde{P_{\chi}}\left(f_{1}\right)=\frac{\zeta(2) L(1 / 2, B C(\pi) \otimes \chi)}{4 L(1, \pi, \operatorname{Ad}) L(1, \mu)^{2}} \alpha\left(f_{1} \otimes \overline{f_{1}}\right)
$$

The global bilinear form

$$
\alpha: \pi \times \tilde{\pi} \rightarrow \mathbb{C}
$$

is the tensor product of the local bilinear forms $\alpha: \pi_{v} \otimes \widetilde{\pi}_{v} \rightarrow \mathbb{C}$ introduced in the last section.

Remark 4.2. The Waldspurger formula above looks slightly different from the original in [Wal85] or from the Waldspurger formula listed in [YZZ13]. Waldspurger chose the global Haar measure used in the period integral such that the $\operatorname{Vol}\left(E^{\times} \backslash \mathbb{A}_{E}^{\times} / \mathbb{A}^{\times}\right)=$ $2 L(1, \mu)$. The authors of [YZZ13] chose the global Haar measure such that the volume is 1 . Then each chose local measures to be compatible with their choice of global measure. With our choice of measures, our formulation is equivalent.

Remark 4.3. Waldspurger assumed the central character of $\pi$ is trivial, but the authors of [YZZ13] removed this condition.

\subsection{Proof of Conjecture 1.3 for $\mathrm{GSpin}_{2} \times \mathrm{GSpin}_{3}$ : Waldspurger Formula Case}

As in the last section, let $F$ be a number field with adele ring $\mathbb{A}_{F}$. Denote $D$ to be the quaternion algebra over $F$ and define $E$ as a quadratic field extension of $F$ with 
fixed embedding $E \hookrightarrow D$ over $F$. Let $\mu: \mathbb{A}_{F}^{\times} / F^{\times} \rightarrow \mathbb{C}$ be the quadratic character associated to the extension $E / F$. Denote the completed zeta function of $F$ as $\xi_{F}(s)$.

As stated in the introduction of this chapter, here we consider the case where $\mathrm{GSpin}_{2}$ is quasi-split, but not split, so $\mathrm{GSpin}_{2} \cong \operatorname{Res}_{E / F} \mathrm{GL}_{1}$ and

$$
\mathrm{GSpin}_{3}= \begin{cases}\mathrm{GL}_{2} & \text { if } D=M_{2}(F) \\ D^{\times} & \text {if } D \text { is non-split. }\end{cases}
$$

The quotient $D^{\times} / \mathrm{GL}_{1}$ can be considered as a special orthogonal group associated to a 3 -dimensional quadratic space over $F$. Recall that $\Delta_{\mathrm{G}_{3}}=\xi_{F}(2)$. Let $T=S O_{2} \cong$ $A_{2} \backslash \mathrm{GSpin}_{2}$ be an anisotropic torus of $A_{3} \backslash \mathrm{GSpin}_{3}$. Let $E$ be a splitting field of $T$ over $F$. One has the following exact sequences:

$$
\begin{gathered}
1 \longrightarrow \mathrm{GL}_{1} \longrightarrow \operatorname{Res}_{E / F} \mathrm{GL}_{1} \longrightarrow T \longrightarrow 1 \\
\left(1 \longrightarrow A_{2} \longrightarrow \mathrm{GSpin}_{2} \longrightarrow \mathrm{SO}_{2} \longrightarrow 1\right)
\end{gathered}
$$

and

$$
\begin{gathered}
1 \longrightarrow \mathrm{GL}_{1} \longrightarrow D^{\times} \longrightarrow D^{\times} / \mathrm{GL}_{1} \longrightarrow 1 \\
\left(1 \longrightarrow A_{3} \longrightarrow \mathrm{GSpin}_{3} \longrightarrow \mathrm{SO}_{3} \longrightarrow 1\right)
\end{gathered}
$$

Let $\pi$ be an irreducible cuspidal automorphic representation of $D^{\times}$with central character $\omega_{\pi}$ and $\chi$ a Hecke character on $\mathbb{A}_{E}^{\times} / E^{\times}$. Note that

$$
\omega_{\pi}: \mathbb{A}_{F}^{\times} / F^{\times} \rightarrow \mathbb{C}^{\times},\left.\quad \chi\right|_{\mathbb{A}_{F}^{\times}}: \mathbb{A}_{F}^{\times} / F^{\times} \rightarrow \mathbb{C}^{\times}
$$

Assume there exists $\omega: \mathbb{A}_{F}^{\times} / F^{\times} \rightarrow \mathbb{C}^{\times}$such that $\omega^{2}=\left.\omega_{\pi} \chi\right|_{\mathbb{A}_{F}^{\times}}$. Then

$$
\left.\left(\omega^{-2} \omega_{\pi}\right) \cdot \chi\right|_{\mathbb{A} \times}=1
$$


Define $\pi \otimes \omega^{-1}$ by $\left(\pi \otimes \omega^{-1}\right)(g)=\pi(g) \omega^{-1}(N(g))$ where $N$ is the norm map

$$
N: D^{\times} \rightarrow \mathbb{A}^{\times} .
$$

Let $z$ be in the center of $D$. Then

$$
\begin{aligned}
\left(\pi \otimes \omega^{-1}\right)(z) & =\pi(z) \omega^{-1}(N(z)) \\
& =\omega_{\pi}(z) \omega^{-1}\left(z^{2}\right) \\
& =\omega_{\pi}(z) \omega^{-2}(z) \\
& =\omega_{\pi} \omega^{-2}(z) .
\end{aligned}
$$

Hence, $\omega_{\pi \otimes \omega^{-1}}=\omega^{-2} \omega_{\pi}$. Using $\pi \otimes \omega^{-1}$ in Waldspurger's formula we obtain

$$
\begin{aligned}
\mathcal{P}\left(f_{\pi}, f_{\chi}\right) & =\frac{\xi_{F}(2) L\left(1 / 2, B C\left(\pi \otimes \omega^{-1}\right) \otimes \chi\right)}{4 L(1, \mu)^{2} L\left(1, \pi \otimes \omega^{-1}, \mathrm{Ad}\right)} \prod_{v} \mathcal{P}\left(f_{\pi, v}, f_{\chi, v}\right) \\
& =\frac{\xi_{F}(2) L\left(1 / 2, B C\left(\pi \otimes \omega^{-1}\right) \otimes \chi\right)}{4 L(1, \chi, \mathrm{Ad}) L(1, \pi, \mathrm{Ad})} \prod_{v} \mathcal{P}\left(f_{\pi, v}, f_{\chi, v}\right)
\end{aligned}
$$

Note that ([Bum97, pg. 102])

$$
L\left(s, B C\left(\pi \otimes \omega^{-1}\right) \otimes \chi\right)=L\left(s, \pi \otimes \omega^{-1} \times \theta_{\chi}\right)
$$

where $\theta_{\chi}$ is the automorphic induction of $\chi$. Hence, one has

$$
\mathcal{P}\left(f_{\pi}, f_{\chi}\right)=\frac{\xi_{F}(2) L\left(1 / 2, \pi \otimes \omega^{-1} \times \chi\right)}{4 L(1, \chi, \mathrm{Ad}) L(1, \pi, \mathrm{Ad})} \prod_{v} \mathcal{P}\left(f_{\pi, v}, f_{\chi, v}\right),
$$

confirming Conjecture 1.3. Moreover, $\left|\mathcal{S}_{\phi_{2}}\right|=\left|\mathcal{S}_{\phi_{3}}\right|=1$, so $2^{\beta}=4\left|\mathcal{S}_{\phi_{2}}\right|\left|\mathcal{S}_{\phi_{3}}\right|$, confirming Conjecture 1.4.

\subsection{Jacquet-Langlands Case}

For the case when $\mathrm{GSpin}_{2}$ is split, then we have $\mathrm{GL}_{1} \times \mathrm{GL}_{1} \subseteq \mathrm{GL}_{2}$. Let $\pi_{2}$ be an irreducible cuspidal automorphic representation of $\mathrm{GL}_{1} \times \mathrm{GL}_{1}$ and set $\pi_{2}=\chi_{1} \otimes \chi_{2}$. 
Let $\pi_{3}$ be an irreducible cuspidal automorphic representation of $\mathrm{GL}_{2}$ with central character $\omega_{\pi_{3}}$. Assume $\omega^{2}=\chi_{1} \chi_{2} \omega_{\pi_{3}}$ and let $\phi$ be in $\pi_{3}$.

First consider the integral,

$$
\begin{aligned}
\int_{\left[\Delta \mathrm{GL}_{1} \backslash\left(\mathrm{GL}_{1} \times \mathrm{GL}_{1}\right)\right]} \phi\left(\begin{array}{ll}
x & \\
& y
\end{array}\right) \chi_{1}(x) \chi_{2}(y) \omega(x y)^{-1} d x d y \\
=\int_{\Delta \mathrm{GL}_{1} \backslash\left(\mathrm{GL}_{1} \times \mathrm{GL}_{1}\right)} \phi\left(\left(\begin{array}{ll}
y^{-1} & \\
& y^{-1}
\end{array}\right)\left(\begin{array}{ll}
x & \\
& y
\end{array}\right)\right) \\
=\int_{\Delta \mathrm{GL}_{1} \backslash\left(\mathrm{GL}_{1} \times \mathrm{GL}_{1}\right)} \phi\left(\begin{array}{ll}
x y_{\pi_{3}}(y) \chi_{1}(x) \chi_{2}(y) \omega(x y)^{-1} d x d y \\
\cdot \omega_{\pi_{3}}(y) \chi_{1}(x) \chi_{2}(y) \omega(x y)^{-1} d x d y
\end{array}\right.
\end{aligned}
$$

Since $\omega_{\pi_{3}}(y) \chi_{2}(y)=\omega^{2}(y) \chi_{1}^{-1}(y)=\omega\left(y^{2}\right) \chi_{1}^{-1}(y)$, we replace in the integral and use [Bum97, pg. 338] to obtain

$$
\begin{aligned}
\int_{\Delta \mathrm{GL}_{1} \backslash\left(\mathrm{GL}_{1} \times \mathrm{GL}_{1}\right)} & \phi\left(\begin{array}{ll}
x y^{-1} & \\
& 1
\end{array}\right) \chi_{1}\left(x y^{-1}\right) \omega\left(x y^{-1}\right)^{-1} d x d y \\
& =\int_{F^{\times} \backslash \mathbb{A}^{\times}} \phi\left(\begin{array}{ll}
z & \\
& 1
\end{array}\right) \chi_{1}(z) \omega(z)^{-1} d z \\
& =Z\left(1 / 2, \pi_{3}, \chi_{1} \omega^{-1}\right) .
\end{aligned}
$$

Now consider the following integral, recalling $\widetilde{\pi}_{3} \cong \pi_{3} \otimes \omega_{\pi_{3}}^{-1}$,

$$
\begin{aligned}
& \int_{\left[\Delta \mathrm{GL}_{1} \backslash\left(\mathrm{GL}_{1} \times \mathrm{GL}_{1}\right)\right]} \overline{\phi\left(\begin{array}{ll}
x & \\
& y
\end{array}\right) \chi_{1}(x) \chi_{2}(y) \omega(x y)^{-1}} d x d y \\
&=\int_{\Delta \mathrm{GL}_{1} \backslash\left(\mathrm{GL}_{1} \times \mathrm{GL}_{1}\right)} \bar{\phi}\left(\begin{array}{rr}
x y^{-1} & \\
1
\end{array}\right) \chi_{1}\left(x y^{-1}\right)^{-1} \omega\left(x y^{-1}\right) d x d y \\
&=\int_{F^{\times} \backslash \mathbb{A} \times} \bar{\phi}\left(\begin{array}{ll}
z & \\
& 1
\end{array}\right) \chi_{1}(z)^{-1} \omega(z) d z \\
&=Z\left(1 / 2, \widetilde{\pi}_{3} \times \chi_{1}^{-1} \omega\right)=Z\left(1 / 2, \pi_{3} \times \omega_{\pi_{3}}^{-1} \chi_{1}^{-1} w\right) \\
&=Z\left(1 / 2, \pi_{3} \times \chi_{2} \omega^{-1}\right) .
\end{aligned}
$$

Per [Wal85, Prop.6], $\mathcal{B}_{\pi_{3}}(\phi, \phi)=2 \zeta(2)^{-1} L\left(1, \mathrm{Ad}, \pi_{3}\right) \prod \mathcal{W}_{\pi_{3, v}}\left(\phi_{v}, \phi_{v}\right)$. Note that $\mathcal{B}_{\pi_{2}}\left(\chi_{1} \otimes \chi_{2}\right)=1$ and $L\left(s, \pi_{2}, \mathrm{Ad}\right)=\zeta(s)^{2}$. Putting this together, one has 


$$
\frac{\mathcal{P}\left(\phi, \chi_{1} \otimes \chi_{2}\right)}{\mathcal{B}_{\pi_{3}}(\phi, \phi) \mathcal{B}_{\pi_{2}}\left(\chi_{1} \otimes \chi_{2}\right)}=\frac{\zeta(2) L\left(1 / 2, \pi_{3} \otimes \omega^{-1} \times \pi_{2}\right)}{2 L\left(1, \mathrm{Ad}, \pi_{3}\right)} \prod_{v} \frac{\mathcal{P}_{v}\left(\phi_{v}, \chi_{1, v} \otimes \chi_{2, v}\right)}{\mathcal{W}_{\pi_{3}}\left(\phi_{v}, \phi_{v}\right) \mathcal{B}_{\pi_{2}}\left(\chi_{1, v} \otimes \chi_{2, v}\right)}
$$

Moreover, $\left|\mathcal{S}_{\phi_{2}}\right|=\left|\mathcal{S}_{\phi_{3}}\right|=1$. Note that we do not have $L\left(s, \pi_{2}, \mathrm{Ad}\right)$ in the denominator, so this case does not confirm Conjecture 1.3. Moreover, $2^{\beta} \neq 4\left|\mathcal{S}_{\phi_{2}}\right|\left|\mathcal{S}_{\phi_{3}}\right|$ and so this case does not appear to confirm Conjecture 1.4 either. 


\section{Bibliography}

[AS06] Mahdi Asgari and Freydoon Shahidi, Generic transfer for general spin groups, Duke Math. J. 132 (2006), no. 1, 137-190.

[AS14] Image of functoriality for general spin groups, Manuscripta Math. 144 (2014), no. 3-4, 609-638.

[Asg02] Mahdi Asgari, Local L-functions for split spinor groups, Canad. J. Math. 54 (2002), no. 4, 673-693.

[Bum97] Daniel Bump, Automorphic forms and representations, Cambridge Studies in Advanced Mathematics, vol. 55, Cambridge University Press, Cambridge, 1997. MR 1431508

[CKM04] James W. Cogdell, Henry H. Kim, and M. Ram Murty, Lectures on automorphic L-functions, Fields Institute Monographs, vol. 20, American Mathematical Society, Providence, RI, 2004.

[Del72] Pierre Deligne, La conjecture de Weil pour les surfaces K3, Invent. Math. 15 (1972), 206-226. MR 0296076

[GGP12] Wee Teck Gan, Benedict H. Gross, and Dipendra Prasad, Symplectic local root numbers, central critical $L$ values, and restriction problems in the 
representation theory of classical groups, Astérisque (2012), no. 346, 1109, Sur les conjectures de Gross et Prasad. I.

[GI11] Wee Teck Gan and Atsushi Ichino, On endoscopy and the refined GrossPrasad conjecture for $\left(\mathrm{SO}_{5}, \mathrm{SO}_{4}\right)$, J. Inst. Math. Jussieu 10 (2011), no. 2, $235-324$.

[GJR05] David Ginzburg, Dihua Jiang, and Stephen Rallis, On the nonvanishing of the central value of the Rankin-Selberg L-functions. II, Automorphic representations, $L$-functions and applications: progress and prospects, Ohio State Univ. Math. Res. Inst. Publ., vol. 11, de Gruyter, Berlin, 2005, pp. 157-191. MR 2192823

[GP92] Benedict H. Gross and Dipendra Prasad, On the decomposition of a representation of $\mathrm{SO}_{n}$ when restricted to $\mathrm{SO}_{n-1}$, Canad. J. Math. 44 (1992), no. $5,974-1002$.

[GP94] - On irreducible representations of $\mathrm{SO}_{2 n+1} \times \mathrm{SO}_{2 m}$, Canad. J. Math. 46 (1994), no. 5, 930-950.

[GPSR97] D. Ginzburg, I. Piatetski-Shapiro, and S. Rallis, L functions for the orthogonal group, Mem. Amer. Math. Soc. 128 (1997), no. 611, viii+218.

[Gro97] Benedict H. Gross, On the motive of a reductive group, Invent. Math. 130 (1997), no. 2, 287-313.

[Har14] R. Neal Harris, The refined Gross-Prasad conjecture for unitary groups, Int. Math. Res. Not. IMRN (2014), no. 2, 303-389. 
[He03] Hongyu He, Unitary representations and theta correspondence for type I classical groups, J. Funct. Anal. 199 (2003), no. 1, 92-121.

[Hel00] Sigurdur Helgason, Groups and geometric analysis, Mathematical Surveys and Monographs, vol. 83, American Mathematical Society, Providence, RI, 2000, Integral geometry, invariant differential operators, and spherical functions, Corrected reprint of the 1984 original. MR 1790156

[Ich08] Atsushi Ichino, Trilinear forms and the central values of triple product L-functions, Duke Math. J. 145 (2008), no. 2, 281-307.

[II10] Atsushi Ichino and Tamutsu Ikeda, On the periods of automorphic forms on special orthogonal groups and the Gross-Prasad conjecture, Geom. Funct. Anal. 19 (2010), no. 5, 1378-1425.

[KMS03] Shin-ichi Kato, Atsushi Murase, and Takashi Sugano, Whittaker-Shintani functions for orthogonal groups, Tohoku Math. J. (2) 55 (2003), no. 1, $1-64$.

[LR05] Erez M. Lapid and Stephen Rallis, On the local factors of representations of classical groups, Automorphic representations, $L$-functions and applications: progress and prospects, Ohio State Univ. Math. Res. Inst. Publ., vol. 11, de Gruyter, Berlin, 2005, pp. 309-359.

[Roh94] David E. Rohrlich, Elliptic curves and the Weil-Deligne group, Elliptic curves and related topics, CRM Proc. Lecture Notes, vol. 4, Amer. Math. Soc., Providence, RI, 1994, pp. 125-157. MR 1260960 
[Sha10] Freydoon Shahidi, Eisenstein series and automorphic L-functions, American Mathematical Society Colloquium Publications, vol. 58, American Mathematical Society, Providence, RI, 2010. MR 2683009

[Sil79] Allan J. Silberger, Introduction to harmonic analysis on reductive p-adic groups, Mathematical Notes, vol. 23, Princeton University Press, Princeton, N.J.; University of Tokyo Press, Tokyo, 1979, Based on lectures by Harish-Chandra at the Institute for Advanced Study, 1971-1973.

[Spr98] T. A. Springer, Linear algebraic groups, second ed., Progress in Mathematics, vol. 9, Birkhäuser Boston, Inc., Boston, MA, 1998.

[Wal85] J.-L. Waldspurger, Sur les valeurs de certaines fonctions L automorphes en leur centre de symétrie, Compositio Math. 54 (1985), no. 2, 173-242.

[Wal03]__ La formule de Plancherel pour les groupes p-adiques (d'après Harish-Chandra), J. Inst. Math. Jussieu 2 (2003), no. 2, 235-333. MR 1989693

[YZZ13] Xinyi Yuan, Shou-Wu Zhang, and Wei Zhang, The Gross-Zagier formula on Shimura curves, Annals of Mathematics Studies, vol. 184, Princeton University Press, Princeton, NJ, 2013. 
Melissa Emory was born in San Jose, California to Duanne Leroy Bosma and Sandra Elaine (Finnie) Bosma. She grew up in Las Vegas, Nevada and has also lived in Germany, Ohio, and Nebraska. Melissa received her B.S. and M.A. in mathematics at the University of Nebraska at Omaha in 2011 and 2013, respectively. Currently, Melissa is a Ph.D. candidate in mathematics at the University of Missouri in Columbia, Missouri where she studies number theory and representation theory under the supervision of Professor Shuichiro Takeda. In July 2018, Melissa begins a position as a Postdoctoral Fellow at the University of Toronto under the supervision of Professor James Arthur. 